\title{
On mixing and transport at a sheared density interface
}

\author{
By GREG D. SULLIVAN'† AND E. JOHN LIST ${ }^{2}$ \\ ${ }^{1}$ Contra Costa Water District, PO Box H2O, Concord, CA 94524, USA \\ ${ }^{2}$ W. M. Keck Laboratory of Hydraulics and Water Resources, California Institute of Technology, \\ Pasadena, CA 91125, USA
}

(Received 22 June 1992 and in revised form 18 February 1994)

Mixing and transport of a stratifying scalar are investigated at a density interface imbedded in a turbulent shear flow. Steady-state interfacial shear flows are generated in a laboratory water channel for layer Richardson numbers, $R i$, between about 1 and 10. The flow field is made optically homogeneous, enabling the use of laser-induced fluorescence with photodiode array imaging to measure the concentration field at high resolution. False-colour images of the concentration field provide valuable insight into interfacial dynamics: when the local mean shear Richardson number, $R i_{s}$, is less than about $0.40-0.45$, interfacial mixing appears to be dominated by Kelvin-Helmholtz $(\mathrm{K}-\mathrm{H})$ instabilities; when $R i_{s}$ is somewhat larger than this, interfacial mixing appears to be dominated by shear-driven wave breaking. In both cases, vertical transport of mixed fluid from the interfacial region into adjacent turbulent layers is accomplished by large-scale turbulent eddies which impinge on the interface and scour fluid from its outer edges.

Motivated by the experimental findings, a model for interfacial mixing and entrainment is developed. A local equilibrium is assumed in which the rate of loss of interfacial fluid by eddy scouring is balanced by the rate of production (local mixing) by interfacial instabilities and molecular diffusion. When a single layer is turbulent and entraining, the model results are as follows: in the molecular-diffusion-dominated regime, $\delta / h \sim P e^{-1 / 2}$ and $E \sim R i^{-1} P e^{-1 / 2}$; in the wave-breaking-dominated regime, $\delta / h \sim R i^{-1 / 2}$ and $E \sim R i^{-3 / 2}$; and in the K-H-dominated regime, $\delta / h \sim R i^{-1}$ and $E \sim$ $R i^{-2}$, where $\delta$ is the interface thickness, $h$ is the boundary-layer thickness, $P e$ is the Péclet number, and $E$ is the normalized entrainment velocity. In all three regimes, the maximum concentration anomaly, $\Gamma_{m} \sim R i^{-1}$. When both layers are turbulent and entraining, $E$ and $\delta$ depend on combinations of parameters from both layers.

\section{Introduction}

Density interfaces occur commonly in a wide variety of flows on various scales. Geophysical (large-scale) examples include haloclines in stratified estuaries, temperature inversions in atmospheric boundary layers, and thermoclines in upper oceanic regions. Engineering (medium and smaller-scale) examples include thermal and wastewater discharges into sizable bays, methane gas flow in coal mines, artificial destratification in reservoirs, and surface-layer flow in solar ponds. In most situations

$\dagger$ Current address: The Chase Manhattan Bank, N.A., 1, Chase Manhattan Plaza, New York, NY 10081, USA. 


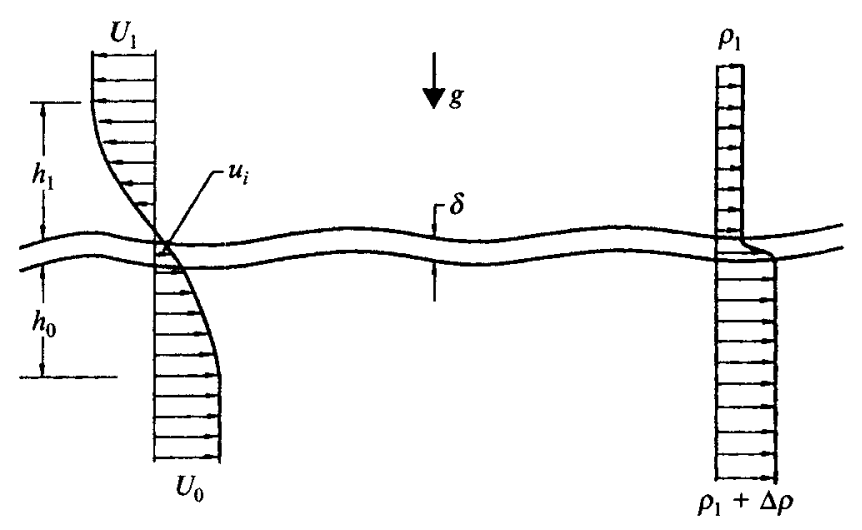

FIGURE 1. Idealized density interface.

of practical interest, characteristic length and velocity scales are quite large, ensuring turbulent flow everywhere except possibly very close to the interface where buoyancy forces can suppress turbulent motions on all scales. Interfacial regions in these flows are typically very sharp, characterized by high gradients of density and velocity.

In this study, attention is restricted to cases in which flow away from the interface is turbulent and buoyancy forces in the interface are sufficiently large that interfacial flow is laminar (except possibly during sporadic periods of instability). These cases comprise an important class of density-stratified shear flows, both because of their practical relevance, and because advances in their understanding represent progress in understanding density-stratified turbulence in general.

An idealized density interface is shown schematically in figure 1. A sharp interface of mean thickness $\delta$ separates fluid of density $\rho_{1}$, above, from fluid of density $\rho_{1}+\Delta \rho$, below. The fluid layers have free-stream velocity $U_{1}$ and $U_{0}$, and boundary-layer thickness $h_{1}$ and $h_{0}$, respectively. (Here $h_{1}$ and $h_{0}$ represent the thicknesses of the turbulent layers; they may be defined in terms of concentration, as done in $\S 3$, but for ease of presentation are depicted in figure 1 in terms of velocity.) The mean interface velocity is $u_{i}$, and gravity acts vertically downward. In this problem, a non-dimensional parameter of fundamental importance is the layer Richardson number,

$$
R i=\Delta b h /\left(U-u_{i}\right)^{2},
$$

representing the relative importance of buoyancy and inertial forces. Here $U$ is the layer free-stream velocity, $h$ is the boundary-layer thickness, and $\Delta b=g \Delta \rho / \rho_{1}$ is the buoyancy jump across the interface. If viscosity and molecular diffusion are important, the layer Reynolds number,

and Péclet number,

$$
\begin{aligned}
& R e=\left|U-u_{i}\right| h / \nu, \\
& P e=\left|U-u_{i}\right| h / \kappa,
\end{aligned}
$$

are also relevant. Here $\nu$ is the kinematic viscosity and $\kappa$ is the scalar diffusivity. If the flow is slowly varying in the streamwise direction, a local equilibrium may be assumed; in this case, the parameters $R i_{0}, R i_{1}, R e_{0}, R e_{1}, P e_{0}$, and $P e_{1}$ determine local properties of the flow.

Of particular interest is the exchange of fluid between the two streams, customarily expressed in terms of an entrainment velocity, $u_{e}$. When a single layer is turbulent and entraining, $u_{e}$ is given by the rate of change in the streamwise direction of the 
volumetric flow rate (per unit span) of the turbulent layer. When both layers are turbulent and entraining, $u_{e}$ is defined in each layer as the volume of equivalent adjacent-layer fluid incorporated per unit area per unit time (Turner 1968). Entrainment is of great importance, as it controls concentrations of scalar quantities such as temperature, oxygen, salt, and pollutants, and affects the global flow structure.

In what follows, previous work relevant to the problem of entrainment at a sheared interface is reviewed. Studies involving additional complicating factors such as rotation and double-diffusive convection are not considered, nor are results from shear-free experiments, such as the grid-generated-turbulence experiments of Hannoun \& List (1988). It is questionable whether results from zero-mean-shear cases are germane to the sheared problem, since properties of the turbulence in the sheared case depend on the shear itself and, more importantly, the mean shear is thought to play an important role in interfacial mixing processes. Fernando (1991) reviews recent work done on the general problem of mixing at a density interface.

Over the past thirty years, sheared density interfaces have been investigated in a number of experimental configurations. To the best of our knowledge, the first studies of entrainment at a density interface involving a mean shearing motion were performed by Ellison \& Turner (1959) on surface jets and on gravity currents flowing down a sloping floor. Shortly thereafter, Lofquist (1960) investigated the flow of a horizontal saline layer beneath ambient fresh water. Following this, Kato \& Phillips (1969) investigated mixing between two fluid layers in an annular tank, the upper layer being driven by an applied surface stress. Next, Moore \& Long (1971) studied the flow of two opposing fluid streams driven by horizontal jets issuing from the floor and ceiling of a racetrack-shaped tank. Later, Kantha, Phillips \& Azad (1977) and Deardorff \& Willis (1982) studied entrainment in annular tank experiments similar to those of Kato \& Phillips. More recently, Narimousa, Long \& Kitaigorodskii (1986) and Stephenson \& Fernando (1991) investigated surface-layer flows in racetrack-shaped tanks, the surface-layer motion in their experiments being driven by a specially designed disc pump.

In each of the above studies, it has been assumed, at least over some region of parameter space, that molecular diffusion of momentum and scalar is unimportant. In this case, the local flow is determined by a single parameter, namely $R i$. Although differences in measured length and velocity scales make absolute comparisons between experiments difficult, similar qualitative trends are observed. At low $R i$, large-scale interfacial disturbances ordinarily observed in equivalent homogeneous flow are absent. Small-scale turbulence still persists, however, at the interface, and adjacent layer fluid is engulfed and incorporated in a manner qualitatively similar to that in equivalent homogeneous flow. As $R i$ is increased, the scale of the turbulence at the interface decreases, and readily identifiable Kelvin-Helmholtz $(\mathrm{K}-\mathrm{H})$ instabilities are observed. At larger $R i, \mathrm{~K}-\mathrm{H}$ instabilities are no longer observed. Interfacial activity appears to be dominated by interfacial waves whose crests are sheared off by turbulent eddies. At even larger $R i$, interfacial wave activity is suppressed, and molecular diffusive effects become important.

Quantitative results from the above experiments are now discussed in terms of normalized entrainment velocity, $E=u_{e} / U$, and layer Richardson number, $R i=$ $\Delta b h / U^{2}$. In each case, the velocity scale, $U$, and length scale, $h$, are related to the freestream velocity and boundary-layer thickness, respectively. Ellison \& Turner studied entrainment for $0<R i<0.8$ and found that $E$ decreased rapidly with increasing $R i$, tending toward zero for values of $R i$ greater than about 0.8 . Unfortunately, they could not obtain accurate estimates of $E$ for values of $R i$ much greater than this, and since 
their experiments were primarily at low $R i$ (turbulent interface), their results are not germane to the present study. Studying a much wider range of $R i, 1<R i<40$, Lofquist found that $E$ decreased monotonically with increasing $R i$, but did not follow a simple power law over the entire range. He also investigated the influence of $R e$ on entrainment, but found no systematic dependence of $E$ on $R e$. Moore \& Long found that $E \sim R i^{-1}$ fit their data reasonably well for $1<R i<30$; however, the introduction of additional length and velocity scales associated with their flow-producing jets may have influenced their results.

Kato \& Phillips scaled their results using the friction velocity, $u_{*}=\left(\tau_{s} / \rho\right)$, where $\tau_{s}$ is the surface stress, and found that a relation of the form $E_{*} \sim R i_{*}^{-1}$ represented their data reasonably well over the full $R i_{*}$ range studied, namely $20<R i_{*}<300$. Here $E_{*}=u_{e} / u_{*}$ and $R i_{*}=\Delta b h / u_{*}^{2}$. Based on their results, they suggested a proportionality between the rate of increase of potential energy of the system and the rate of dissipation of turbulent kinetic energy in the mixed layer. Studying a wider range of $R i_{*}, 30<$ $R i_{*}<1000$, Kantha $e t$ al. later found no simple power law dependence of $E_{*}$ on $R i_{*}$. Phillips (1977) then suggested that the relevant scaling velocity was the mean difference in velocity between the two layers, since shear-flow instabilities at the density interface appeared to initiate the entrainment process. With this in mind, Price (1979) attempted to re-scale the annulus experiments of Kato \& Phillips, and Kantha et al. by using a momentum balance and assuming a quasi-equilibrium $R i \sim 0.6$. He suggested $E \sim$ $R i^{-4}$ for $0.5<R i<1$; however, results from the experiments of Deardorff \& Willis questioned the validity of the quasi-equilibrium $R i$ assumption and further suggested that viscous diffusion of momentum was important, inasmuch as it reduced the velocity difference across the interface. Scaling their results using both $U$ and $u_{*}$, Deardorff \& Willis suggested $E_{*} \sim R i^{-1.4} R i_{*}^{-1 / 2}$.

More recently, Narimousa \& Fernando (1987) analysed the experiments of Narimousa et al. and suggested $E \sim R i^{-1}$ for $0.5<R i<25$. They also found normalized r.m.s. wave amplitudes proportional to $R i^{-1 / 2}$ and, based on this, suggested that the energy gained by interfacial disturbances was proportional to the energy of wave-generating large-scale eddies. Finally, using laser-induced fluorescence and video imaging to measure interface thicknesses, Stephenson \& Fernando suggested $\delta / h \sim R i^{-1}$ for $R i$ less than about 5 and $\delta / h \sim$ constant for $R i$ greater than about 5 .

As the results above show, there is currently no agreement on the form of an entrainment relation. Experimental peculiarities make comparisons difficult, especially in the absence of a theoretical model to suggest the basis on which experimental results should be compared. Most needed is a self-consistent model for entrainment, based on physical principles, that can be (critically) verified experimentally.

The remaining sections are devoted to achieving this end. In $\S 2$, the experimental facility used in this study is briefly described, and in $\$ 3$ results obtained from five experiments are presented. In $\$ 4$, a model for entrainment is developed and model predictions are compared with experimental results. Finally, in $\S 5$, present results are discussed in the context of previous work.

\section{The experiments}

The experiments described herein consist of measurements of tracer dye concentration in two-layer density-stratified shear flows. Details of the two-layer flow facility and laser diagnostics are discussed in the companion paper, Sullivan \& List (1993). A brief synopsis is given here.

The flow generation system is shown schematically in figure 2. Inlets and outlets, 


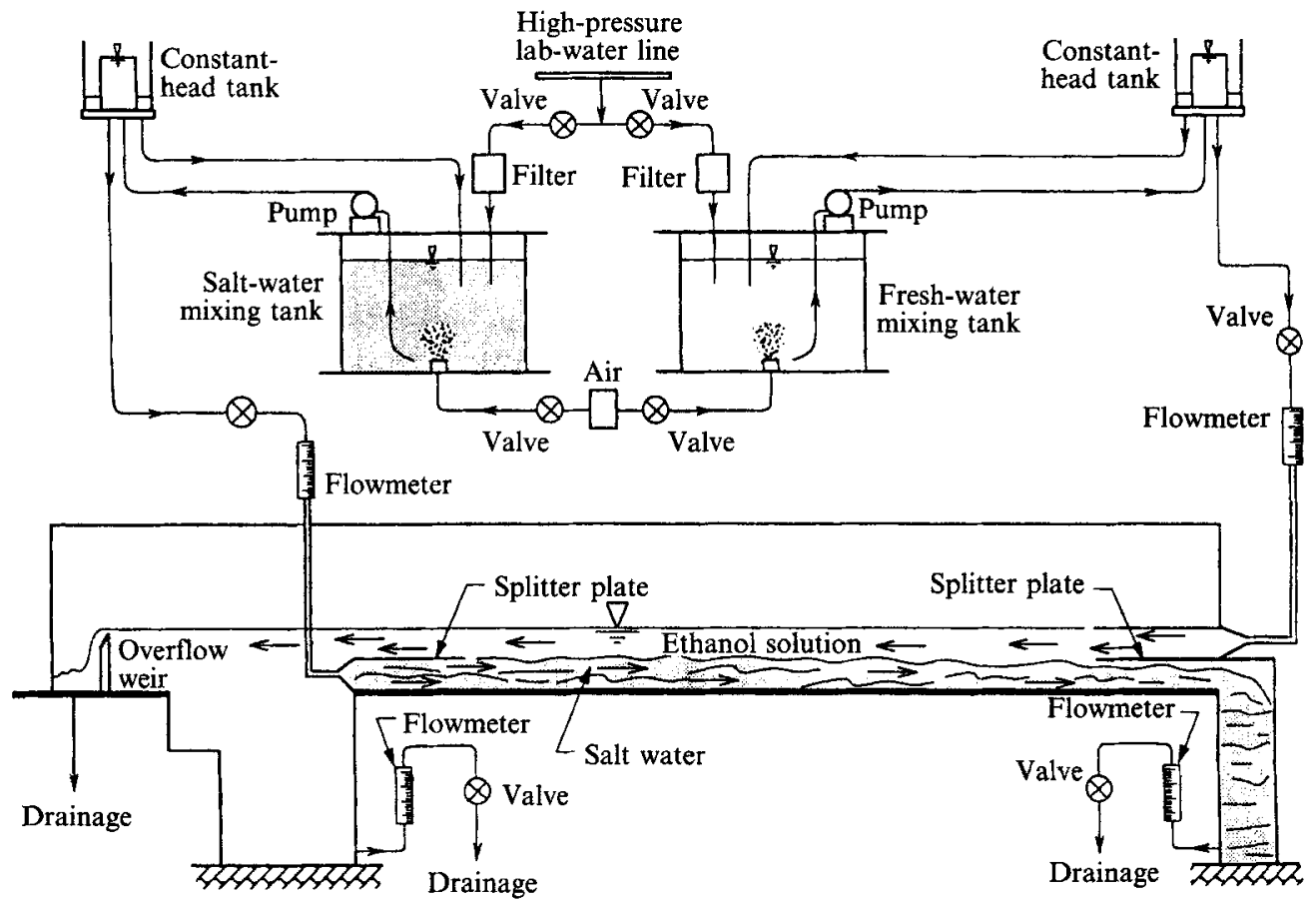

FIGURE 2. Schematic of flow generation system.

separated by splitter plates, have been added to each end of a channel originally designed by Rasi (1989), enabling the generation of steady-state two-layer counterflows over a range of conditions. The length of the channel between the two inlets is approximately $5 \mathrm{~m}$ and the channel width is $10 \mathrm{~cm}$. Aqueous saline solution acts as the heavy fluid and aqueous ethanol solution acts as the light fluid. The two fluids are index-of-refraction matched using the method developed by Hannoun (1985) to facilitate the use of laser-based diagnostics. The saline and ethanol solutions are pumped up from large reservoirs to constant-head tanks, as shown, and flow through valve-flowmeter combinations before entering the flume through the inlet sections. The ethanol solution flows above the saline solution and exits the flume either through the lower outlet or over the sharp-crested overflow weir which maintains a constant total depth of fluid. The saline solution exits through the lower outlet shown.

Laser-induced fluorescence (LIF) is used to measure the concentration of tracer dye, pre-mixed with the lower layer fluid. The method employed is essentially that used successfully by Koochesfahani (1984), Papantoniou (1986), Hannoun (1987), and Rasi (1989). A schematic of the LIF optical layout is shown in figure 3 . The $514.5 \mathrm{~nm}$ line (green) of a $2 \mathrm{~W}$ Argon-ion laser (Spectra Physics 265) is focused near the fluid interface using a series of mirrors and lenses. When excited by laser light of wavelength $514.5 \mathrm{~nm}$, the tracer dye, Rhodamine 6G, fluoresces to emit light centred at approximately $570 \mathrm{~nm}$ (yellow). The emitted light is focused onto an array of 1024 light-sensitive photodiodes housed in a camera assembly (EG \& G Reticon LC300A), as shown. An optical filter is placed in front of the camera's focusing lens, effectively blocking the green laser light and passing the yellow light emitted by the excited dye. The entire array of 1024 photodiodes is sampled approximately 50 times per second, providing detailed information about interfacial mixing and transport.

The dye concentration is determined using an optical transfer function similar to 


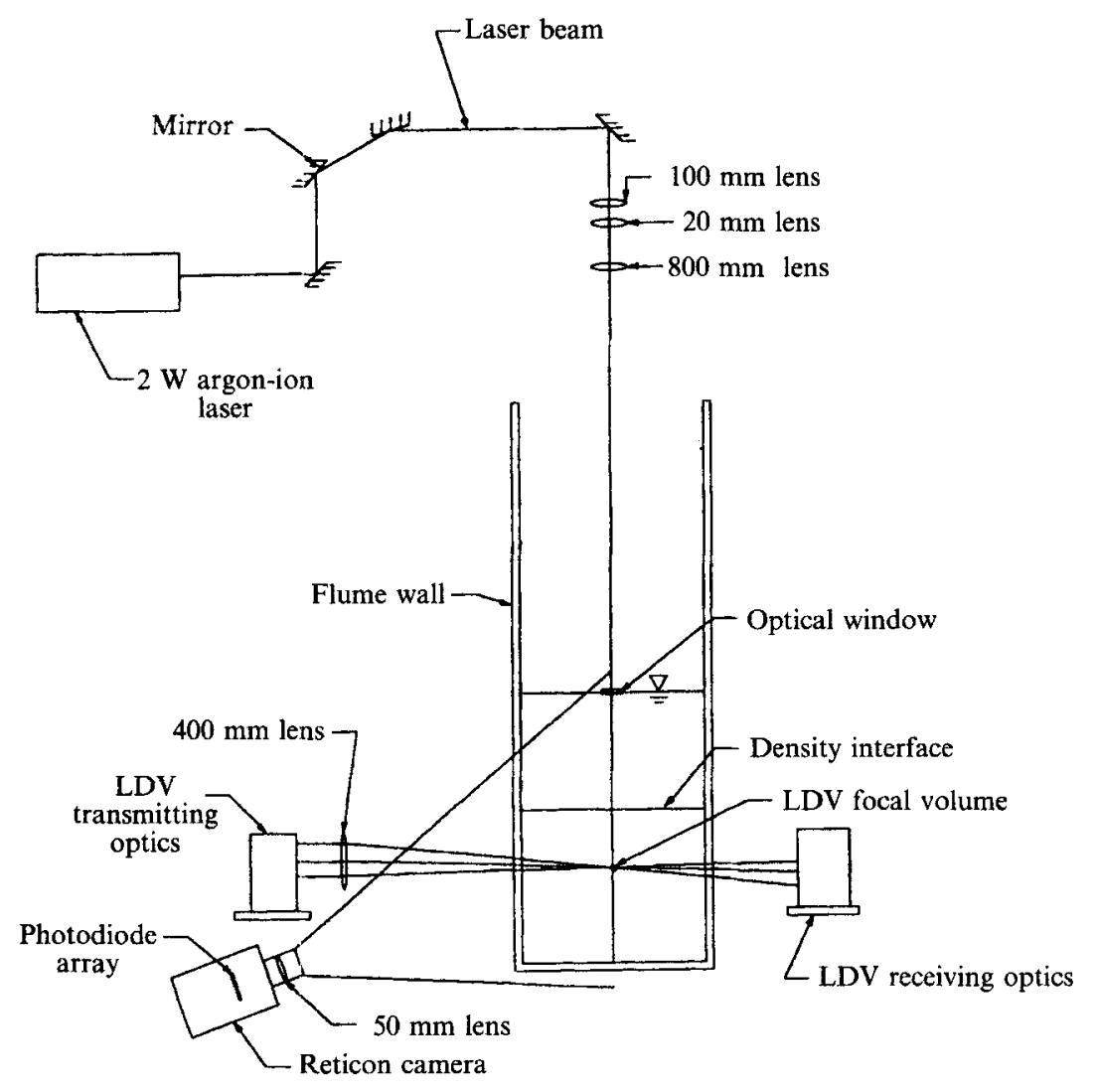

Figure 3. Schematic of LIF optical layout.

that used by Koochesfahani (1984). The optical transfer function corrects for variable beam width, pixel response non-uniformity, beam attenuation by the dye, nonuniformity of the imaging system, and slight array misalignment with the laser beam. Corrections are made for laser power fluctuations on a scan by scan basis by normalizing by the near floor concentration. (In each case, the near floor concentration is essentially that of pure unmixed lower-layer fluid.) The (static) spatial resolution of the LIF measurements is typically $300 \mu \mathrm{m} \times 300 \mu \mathrm{m} \times 400 \mu \mathrm{m}$ per pixel, the first two dimensions corresponding to the field of view of the imaging system, the last dimension corresponding to the laser beam width in the spanwise direction. For further details pertaining to the LIF system, see Sullivan \& List (1993).

Before an experiment, the channel is filled with $19.25 \mathrm{~cm}$ of saline solution, and the LIF system is aligned and calibrated. The channel is then partially drained, and ethanol. solution is added to approximately $10 \mathrm{~cm}$ of saline solution until the total depth of fluid in the channel is $19.25 \mathrm{~cm}$. The inlet and the outlet flow rates of saline solution and ethanol solution are then slowly increased to predetermined levels, and the flow is allowed to equilibrate for 10 to 15 minutes. Once equilibrium is established, the experiment begins and a series of 5120 photodiode array scans are recorded using a Masscomp SLS-5450-01 (Scientific Laboratory) data acquisition system. Immediately following this, velocity measurements are made using a laser-Doppler velocimetry system at a number of positions along the vertical Argon-ion laser beam path until the reservoir fluids are exhausted. (Velocity measurements from the experiments are 
reported in Sullivan \& List 1993.) The channel is then flushed with chlorinated water and the background noise is measured.

\section{Concentration measurements}

Results from five experiments are presented here, including profiles of mean concentration, false colour images of concentration, probability distribution functions of concentration, maximum concentration anomalies, power-spectral-density estimates for concentration, and interface thicknesses.

Local governing parameters are given in table 1 . Here $d$ is the depth of the lower layer and $h$ is the concentration 'boundary-layer' thickness, defined as the distance from the mean interface position, $\bar{\eta}$, to the location above (or below) the interface where the probability of finding fluid differing in concentration from that of the local mean by greater than $0.01 C_{0}$ is 0.02 . (Physically this is a measure of the level to which scalar is transported vertically by turbulent motions. Alternative measures of $h$ have been considered, including momentum thickness, displacement thickness, and thickness based on mean or r.m.s. concentration; after some exploration, it is found that a definition of $h$ based on concentration probability is the least sensitive to measurement errors.) $U$ is the ratio of the volumetric flow rate to cross-sectional area; $u_{i}$ is the mean horizontal velocity at the interface, estimated by linear interpolation; $h_{s}$ is the maximum velocity gradient thickness, defined by

$$
h_{s}=\frac{\left(U_{1}-U_{0}\right)}{(\partial u / \partial y)_{\max }},
$$

where $(\partial u / \partial y)_{\max }$ is the maximum mean velocity gradient at the interface; $R i_{s}$ is the mean shear Richardson number, defined by

$$
R i_{s}=\frac{\Delta b h_{s}}{\left(U_{0}-U_{1}\right)^{2}}
$$

and $R i$ is the layer Richardson number, defined by (1.1) with the mean layer velocity replacing the free-stream velocity. (This is necessary as accurate estimates of maximum layer velocity could not be determined. This does, however, introduce some uncertainty in $R i$, since the ratio of layer maximum to mean velocity depends on the relative widths of the boundary layers.)

In figure 4 , normalized mean concentration, $\left(c_{\infty}-\bar{c}\right) / \Delta C$, is shown near the interface in the lower layer; $c_{\infty}$ is the mean concentration at the outer edge of the concentration boundary layer. Here concentration is measured in a frame of reference moving with the interface to filter out the effect of interfacial waves. Distance below the interface, $\eta-y$, is normalized by the interfacial half-width, $\delta_{0}$, defined as the vertical distance between the centre of the interface and the point below where $c$ first rises above $0.9 \Delta C$. In figure 4 interfacial instabilities strongly influence the form of the profiles: the zone of rapidly varying concentration is confined to a region about one interfacial halfwidth from the interface at $R i_{s}=0.46$ and 0.57 , while at $R i_{s}=0.18,0.25$, and 0.40 , this zone extends out 3 to 4 interfacial half-widths from the interface.

False colour images of concentration in $(t, y)$ space from four experiments are shown in figure $5(a-e)$ (plates 1 and 2). Here $t$ is time and $y$ is vertical position (zero references are taken arbitrarily). The colours correspond to difference $c / C_{0}$ values, given in the caption, where $c$ is the instantaneous dye concentration and $C_{0}$ is the unmixed dye concentration in the lower layer. 


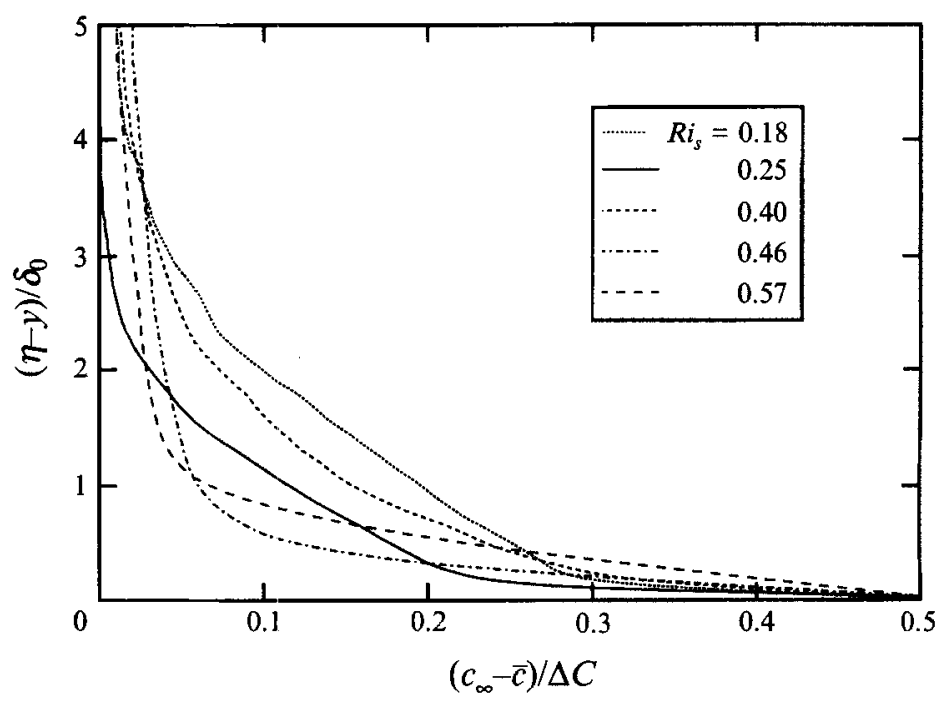

FIGURE 4. Mean concentration in a frame of reference moving with the interface. Values are from the lower layer.

\begin{tabular}{ccccccccccc}
\hline & $\begin{array}{c}d \\
\operatorname{Exp}\end{array}$ & $\begin{array}{c}h_{0} \\
(\mathrm{~cm})\end{array}$ & $\begin{array}{c}h_{1} \\
(\mathrm{~cm})\end{array}$ & $\begin{array}{c}h_{s} \\
(\mathrm{~cm})\end{array}$ & $\begin{array}{c}U_{0} \\
\left(\mathrm{~cm} \mathrm{~s}^{-1}\right)\end{array}$ & $\begin{array}{c}U_{1} \\
\left(\mathrm{~cm} \mathrm{~s}^{-1}\right)\end{array}$ & $\begin{array}{c}u_{i} \\
\left(\mathrm{~cm} \mathrm{~s}^{-1}\right)\end{array}$ & $R i_{0}$ & $R i_{1}$ & $R i_{s}$ \\
1 & 6.17 & 4.14 & 4.66 & 2.93 & 6.89 & -2.97 & 3.34 & 6.19 & 2.20 & 0.57 \\
$4 \dagger$ & 7.10 & - & 4.77 & 1.55 & 5.73 & -3.29 & 1.22 & - & 3.10 & 0.25 \\
10 & 10.57 & 6.12 & 4.45 & 2.22 & 3.89 & -4.59 & -0.04 & 5.09 & 2.75 & 0.40 \\
11 & 11.83 & 4.78 & 3.20 & 1.17 & 3.47 & -3.56 & 0.78 & 4.95 & 1.27 & 0.18 \\
13 & 8.13 & 4.84 & 4.31 & 1.80 & 4.99 & -3.58 & 1.98 & 10.13 & 2.64 & 0.46
\end{tabular}

$\dagger$ The lower layer in experiment 4 is non-turbulent; hence $h_{0}$ and $R i_{0}$ are undefined.

TABLE 1. Local governing parameters.

In all of the flows studied, large-scale turbulent eddies appear to scour fluid from the interface into adjacent layers $\dagger$, as seen, for example, in figure 5(c), event labelled A. Once in an adjacent layer, interfacial fluid presumably becomes permanently incorporated through the combined action of turbulent straining (stretching marked fluid into thin strands) and molecular diffusion. From visual observations it is speculated that if the scoured fluid is too heavy, it may fall back to the interface (i.e. is not incorporated) and produce an interfacial disturbance. In none of the experiments do turbulent eddies entrain pure unmixed fluid directly from an adjacent layer; rather, they always entrain fluid of intermediate density from the interfacial region. Rough estimates show that the length (in time) of a scouring 'event' at a fixed position is typically $\tau_{L}$ to $2 \tau_{L}$ and the time between events at a fixed position is typically $5 \tau_{L}$ to $10 \tau_{L}$, independent of $R i$. Here $\tau_{L}=h / U$ is the large-scale time, where $h$ is the concentration boundary-layer thickness and $U$ is the average layer velocity. An

$\dagger$ In figure $5(a-c)$ scouring events are more clearly observed above the interface than below. This is an artifact of the asymmetric colour thresholding used and not a manifestation of a physical asymmetry. Absolute errors in concentration above the interface are considerably less than those below; asymmetric colour thresholding is employed to reduce visible noise in the lower layer. 


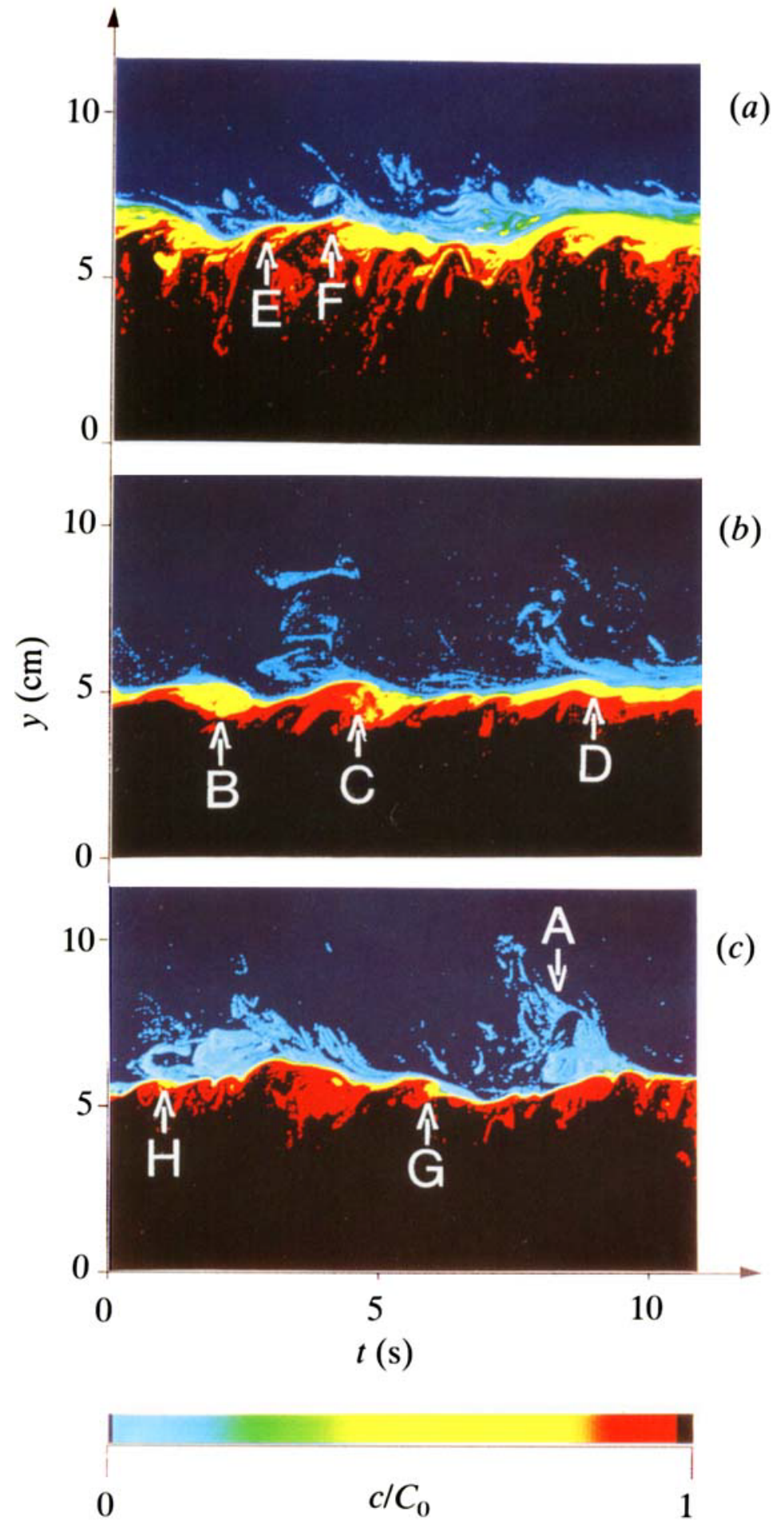

Figure 5. (a-c). For caption see overleaf. 

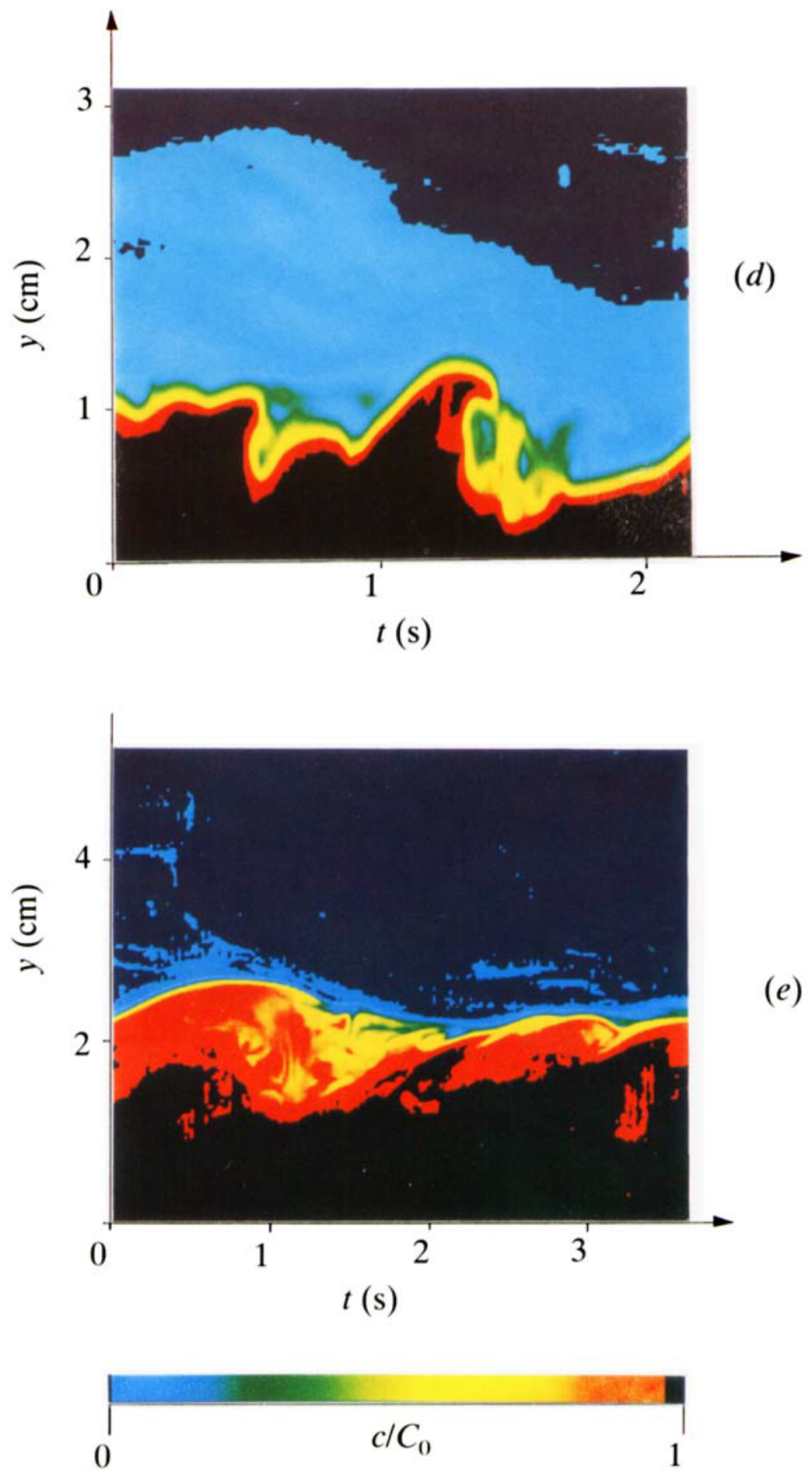

Figure 5. (a-c) False colour images of concentration: (a) $R i_{s}=0.18 ;(b) R i_{s}=0.25 ;(c) R i_{s}=0.46$. Dark blue corresponds to $c / C_{0}<0.015$; light blue to $0.015<c / C_{0}<0.19$; green to $0.19<c / C_{0}<0.47$; yellow to $0.47<c / C_{0}<0.75$; red to $0.75<c / C_{0}<0.97$; and black to $c / C_{0}>0.97$. $(d, e)$ False colour images of concentration: $(d)$ close-up of a wave-breaking event at $R i_{s}=0.57 ;(e)$ close-up of a Kelvin-Helmholtz instability at $R i_{\text {s }}=0.25$. 
examination of estimates of the time of occurrence of scouring events and interfacial instabilities suggests that in all cases these two distinct events are uncorrelated in time.

Although eddy scouring events are similar in the experiments studied, local interfacial mixing appears to take one of two qualitatively different forms. When $R i_{s}$ is below about $0.4-0.45$, large-scale $\mathrm{K}-\mathrm{H}$ instabilities appear at the interface and dominate interfacial mixing. When $R i_{s}$ is somewhat greater than this, shear-driven interfacial wave breaking appears to dominate mixing.

In figure $5(b)\left(R i_{s}=0.25\right), \mathrm{K}-\mathrm{H}$ instabilities at various stages of growth can be seen. The event labelled $\mathrm{B}$ depicts $\mathrm{K}-\mathrm{H}$ instability which has presumably evolved into a turbulent billow. This results in a relatively large patch of mixed fluid at the interface which ultimately will flow as an intrusion along the interface. The event labelled $\mathrm{C}$ depicts the initial stages of roll-up of a $\mathrm{K}-\mathrm{H}$ instability. Note the very sharp interface separating upper and lower fluids in the vicinity of the roll-up. Figure 5(e) shows a close-up of this event. Event D depicts mixed fluid from a $\mathrm{K}-\mathrm{H}$ billow which has presumably collapsed. The intermediate-density fluid (seen here as yellow) generated during the $\mathrm{K}-\mathrm{H}$ roll-up and billowing process flows along the interface seeking its equilibrium density level. In figure $5(a)\left(R i_{s}=0.18\right)$, more intense and more frequently occurring $\mathrm{K}-\mathrm{H}$-type shear instabilities are observed. Events labelled $\mathrm{E}$ and $\mathrm{F}$ depict intense $\mathrm{K}-\mathrm{H}$-type shear instabilities which mix upper- and lower-layer fluid at the interface, locally generating fluid of intermediate density (seen here as yellow-green). At $R i_{s}=0.40$ (not shown), $\mathrm{K}-\mathrm{H}$ instabilities are also observed. The $\mathrm{K}-\mathrm{H}$ instabilities at low $R i_{s}(0.18,0.25,0.40)$ appear to be cyclically generated: they form, roll-up into billows, grow, collapse, and after some period of time they form again. In figure $5(b)$, $\mathrm{K}-\mathrm{H}$ billows are markedly asymmetric with respect to the density interface (e.g. event B). In this case, billows form almost entirely below the centre of the interface, the upper half of the interface remaining remarkably sharp. Such asymmetries can be induced by a displacement between the centre of the density interface and the region of maximum shear (Lawrence, Browand \& Redekopp 1991).

In figure $5(c)\left(R i_{s}=0.46\right), \mathrm{K}-\mathrm{H}$ instabilities are no longer observed; the dominant interfacial mixing mechanism appears to be shear-driven finite-amplitude wave breaking. Interfacial waves, presumably generated by random pressure fluctuations induced by turbulent eddies, appear to be distorted by the mean shear until they become gravitationally unstable and break. Event $\mathrm{G}$ depicts a wave-breaking event at the onset of convective (gravitational) instability. The essential difference between this instability and those in figures $5(a)$ and $5(b)$ is the greatly reduced amount of mixed fluid generated in the gravitational instability. (It should be borne in mind when comparing events such as $\mathrm{E}$ and $\mathrm{G}$ in figures $5(a)$ and $5(c)$ that these are essentially 'snapshots' of mixing events; although they may appear instantaneously to be of comparable extent in the vertical, the appearance of the interface suggests that a much greater amount of mixed fluid is generated on average in events such as E.) The event labelled $\mathrm{H}$ depicts a small patch of mixed fluid presumably generated by a wave breaking event; this presumably spreads out as an intrusion along the interface. At $R i_{s}=0.57$ (not shown), shear-driven wave breaking is also observed. Figure $5(d)$ shows a close-up of a wave breaking event at $R i_{s}=0.57$. The variation (in time) of the interface thicknesses in figure $5(a-c)$ may be attributed to the random nature of the interfacial instabilities and local scouring events. It appears from false colour images such as those shown in figure $5(a-c)$ that regions along the interface away from interfacial instabilities are laminar, as evidenced by the well-defined relatively smooth interface concentration contours.

It is interesting to compare the above observations to those in the experiments of 
Lawrence et al. (1991), Koop \& Browand (1979), and Browand \& Winant (1973). In the earlier experiments the development of instabilities at an initially laminar sheared density interface is investigated when the vertical scale of the shear is considerably greater in extent than that of the density stratification. The previous investigators observed growth and collapse of $\mathrm{K}-\mathrm{H}$ instabilities at low values of $R i_{s}$, as in the present experiments, and a change in the character of the interfacial instabilities as $R i_{s}$ is increased. In their experiments the dominant instability at larger $R i_{s}$ is termed a Hölmböe instability, an inviscid mechanism active when the shear-layer thickness is much larger than the thickness of the density interface. The Hölmböe instabilities observed in the previous experiments, however, involve relatively small growth rates for $R i_{s}$ in the range studied here; and although they may be active in the present experiments, Hölmböe instabilities are thought to be of secondary importance relative to eddy-generated wave breaking. In the present experiments turbulence in adjacent layers on either side of the interface (which is absent in the previous experiments) 'forces' the interface in a highly nonlinear way: large eddies in adjacent layers interact with the density interface, generating finite-amplitude waves which are distorted by the mean shear and break relatively quickly; the large eddies also sharpen the interface which presumably enables $\mathrm{K}-\mathrm{H}$ instabilities to recur after their collapse.

The distinction made between 'wave-breaking' instabilities and $\mathrm{K}-\mathrm{H}$ instabilities in the foregoing is now discussed. Wave breaking events are essentially a convective instability resulting from the interaction of the mean shear with finite-amplitude interfacial waves. The finite-amplitude waves may be generated by turbulence-induced pressure fluctuations in turbulent layers adjacent to the interface. In the absence of turbulence in an adjacent layer, these instabilities presumably do not occur. $\mathrm{K}-\mathrm{H}$ instabilities, on the other hand, result when the destabilizing effect of the shear in the interfacial region is large enough to overcome the stabilizing effect of the buoyancy gradient. The $\mathrm{K}-\mathrm{H}$ shear instabilities do not require turbulence in an adjacent layer. In the case of a wave-breaking event (convective instability), visual observations suggest that the 'breaking" or overturning process generates a small patch of mixed fluid at the interface which does not grow with time. In contrast, in the case of a $\mathrm{K}-\mathbf{H}$ shear instability, visual observations suggest that a turbulent patch of mixed fluid is generated at the interface which does grow with time until, presumably, turbulence can no longer be locally sustained within the patch. Thus, the vertical scale of the wavebreaking event is typically much smaller than that of the $\mathrm{K}-\mathrm{H}$ instability and the two can be distinguished by markedly different mean and r.m.s. concentration profiles (Sullivan \& List 1993).

In the previous discussion, the mean-shear Richardson number, $R i_{s}$, is used to characterize interfacial instability. It should be noted that $R i_{s}$ is related to the mean gradient Richardson number in the interface by $R i_{s} \sim R i_{g}\left(\delta / h_{s}\right)$, where

$$
R i_{g}=\frac{-(g / \rho) \partial \rho / \partial y}{(\partial u / \partial y)^{2}} \sim \frac{\Delta b h_{s}^{2}}{(\Delta U)^{2} \delta} .
$$

When $\left(\delta / h_{s}\right) \ll 1$, results from Lawrence et al. (1991) suggest that $R i_{s}$ is the relevant interfacial stability parameter. And since estimates of $R i_{s}$ involve one less experimentally measured parameter than $R i_{g}, R i_{s}$ is chosen here to characterize stability at the interface. (It should be noted that measured values of $R i_{s}$ in the present experiments have an estimated uncertainty of about $20 \%$; the range of $R i_{s}$ suggested in the present experiments for transition between $\mathrm{K}-\mathrm{H}$ and wave-breaking-dominated mixing at the interface is only approximate.)

A typical concentration-time trace measured a distance $\frac{1}{2} h_{1}$ above the interface is 


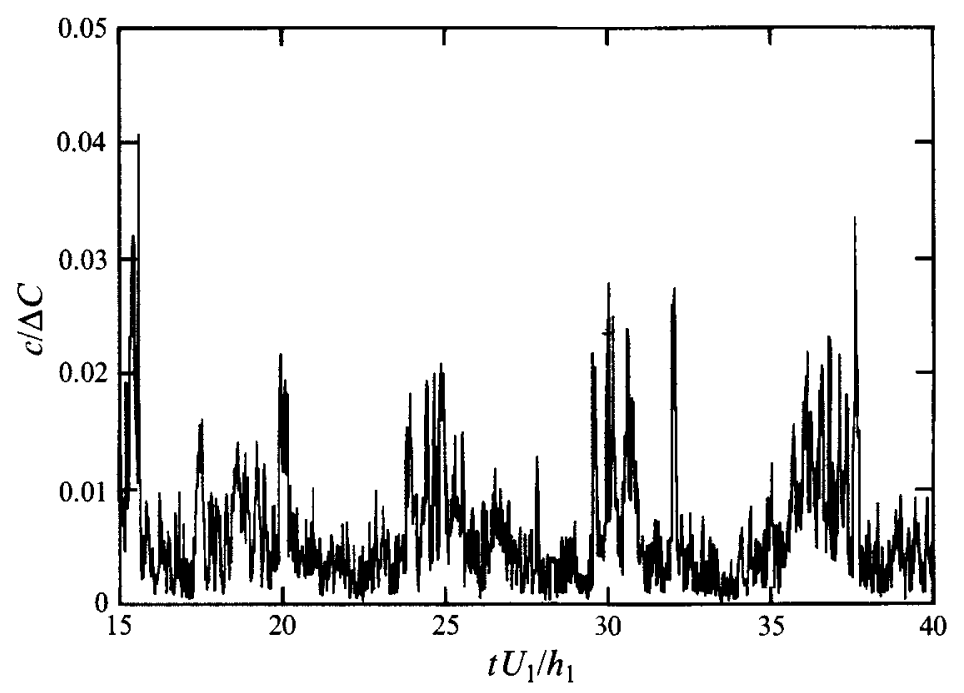

FIGURE 6. Concentration fluctuations at $(y-\eta) / h_{1}=0.5 .18 .1<t U_{1} / h_{1}<27.2$ corresponds to the false colour image segment shown in figure $5(c)$. Here $\Delta C$ is the concentration jump across the interface and $\eta$ is the interface position.

shown in figure 6 . Here time is normalized by the large-scale time, $h_{1} / U_{1}$, where $U_{1}$ is the upper-layer average velocity and $h_{1}$ is the upper-layer concentration-boundary-layer thickness. Measurements are taken in a frame of reference moving with the interface to remove wave effects. The region $18.1<t U_{1} / h_{1}<27.2$ corresponds to the false colour image segment shown in figure $5(\mathrm{c})$. Eddy scouring events are manifested in figure 6 as regions of relatively high concentration fluctuations, typically spanning 1 to 2 large-scale times and spaced by about 5 to 7 large-scale times. During a scouring event, the concentration signal is highly intermittent; regions of scoured interfacial fluid of intermediate concentration are interspersed between regions of nearly pure unmixed upper-layer fluid. Note in figure 6 that the highest concentrations observed a distance $\frac{1}{2} h_{1}$ above the interface are only about $4 \%$ of the concentration in the lower layer. It should also be borne in mind that when comparing figure 6 to figure $5(\mathrm{c})$, fluid with concentration in the range $0 \leqslant c / \Delta C<0.015$ is shown in figure $5(c)$ as dark blue; i.e. any fluctuations with $c / \Delta C<0.015$ in figure 6 will not be observed in figure $5(c)$, since these are below the $c / \Delta C=0.015$ threshold. (The threshold is set to reduce noise in the visual colour images.)

Probability distribution functions of dye concentration anomaly are shown in figures $7(a)$ and $7(b)$. (Strictly speaking these are plots of $1-P$, where $P$ is the traditionally defined probability distribution function.) The probability of finding normalized concentration anomaly, $|\bar{c}-c| / \Delta C$, greater than $\Gamma$ is plotted for a point located a distance $\frac{1}{2} h_{0}$ below the interface (figure $7 a$ ) and for a point located a distance $\frac{1}{2} h_{1}$ above the interface (figure $7 b$ ). Here $\bar{c}$ is the time-averaged local concentration, $\Delta C$ is the concentration jump across the interface, and $h_{0}$ and $h_{1}$ are the concentration-boundarylayer thicknesses in the lower and upper layer, respectively. Measurements are taken in a frame of reference moving with the interface to remove wave effects. In figure $7(a)$, only four curves are shown; in the case of experiment $4\left(R i_{s}=0.25\right)$, the lower layer is non-turbulent and the probability distribution function of concentration anomaly is not pertinent. Note that the distribution functions in figure 7 do not display a simple $R i$ dependence over the range of $\Gamma$ shown. 

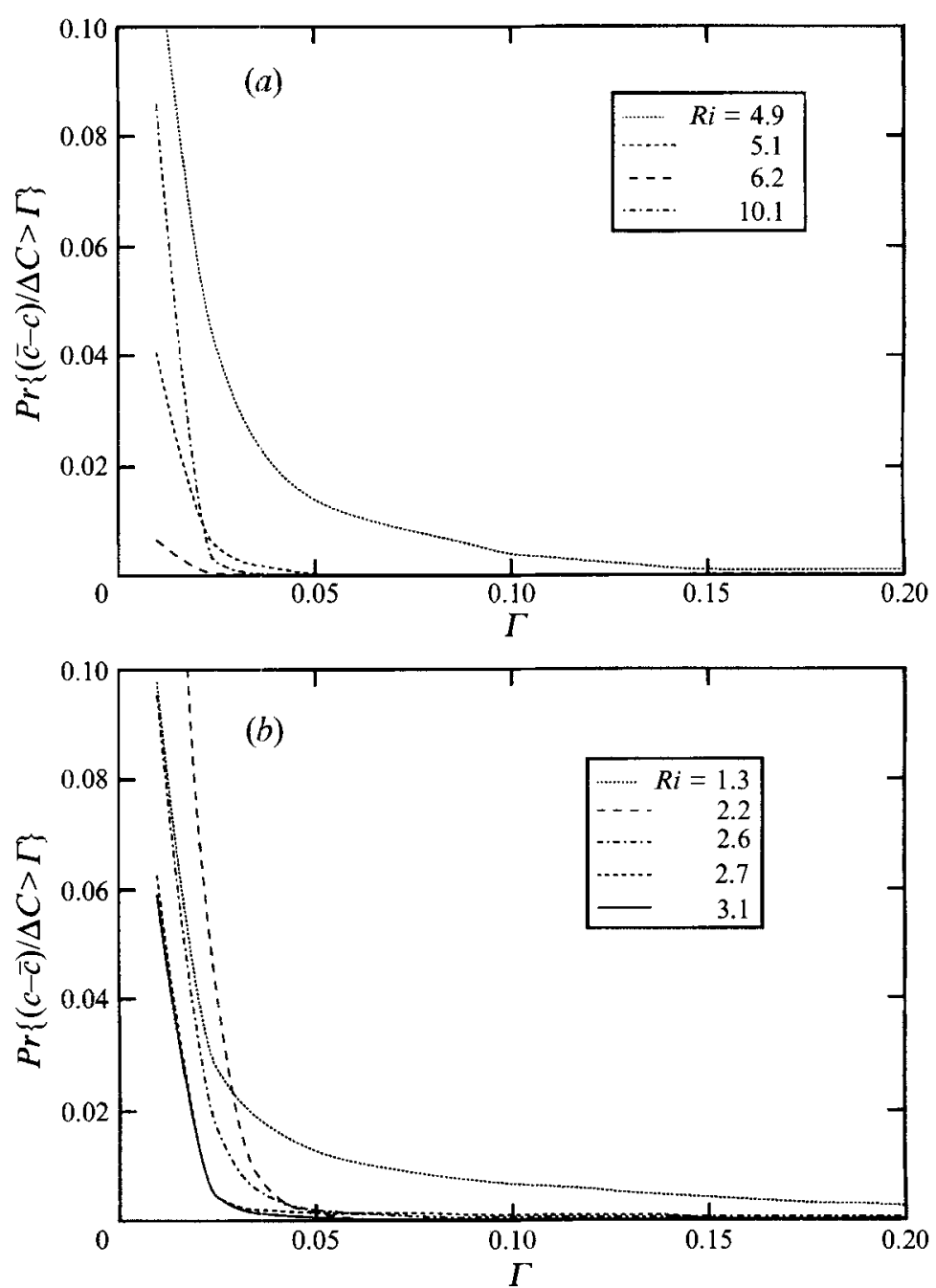

FIGURE 7. Probability distribution function of concentration anomaly. Measurements are taken in a frame of reference moving with the interface at $(a)$ a position $(\eta-y) / h_{0}=0.5$ below the interface, and (b) a position $(y-\eta) / h_{1}=0.5$ above the interface. Note that the $R i_{s}=0.25$ experiment is not included in $(a)$; the lower layer is non-turbulent in this case.

From the distribution function, the maximum (normalized) concentration anomaly is defined as the concentration, $\Gamma_{m}$, for which

$$
\operatorname{Pr}\{|c-\bar{c}| / \Delta C\}>\Gamma_{m}=K
$$

at $|y-\eta| / h=0.5$; i.e. the probability of finding concentration anomaly, $|c-\bar{c}| / \Delta C$, in excess of $\Gamma_{m}$ is $K$, the probability threshold. There is some arbitrariness in the selection of $K$; however, variations in $K$ do not significantly alter the nature of the results so long as $K$ is sufficiently small. Figure 8 shows $\Gamma_{m}$ as a function of $R i$ for $K=0.01$. Error bars shown indicate maximum probable deviations from measured values. The error in $R i$ arises primarily from errors in estimates of $h$ and $u_{i}$. The error in $\Gamma_{m}$ is estimated as $\pm \frac{1}{2}$ bin range in figure 7 (probabilities are calculated in figure 7 at intervals of $\Gamma$ of 0.01 , which gives an estimated error of \pm 0.005 ). $\Gamma_{m}$ in figure 8 is intended to represent the 


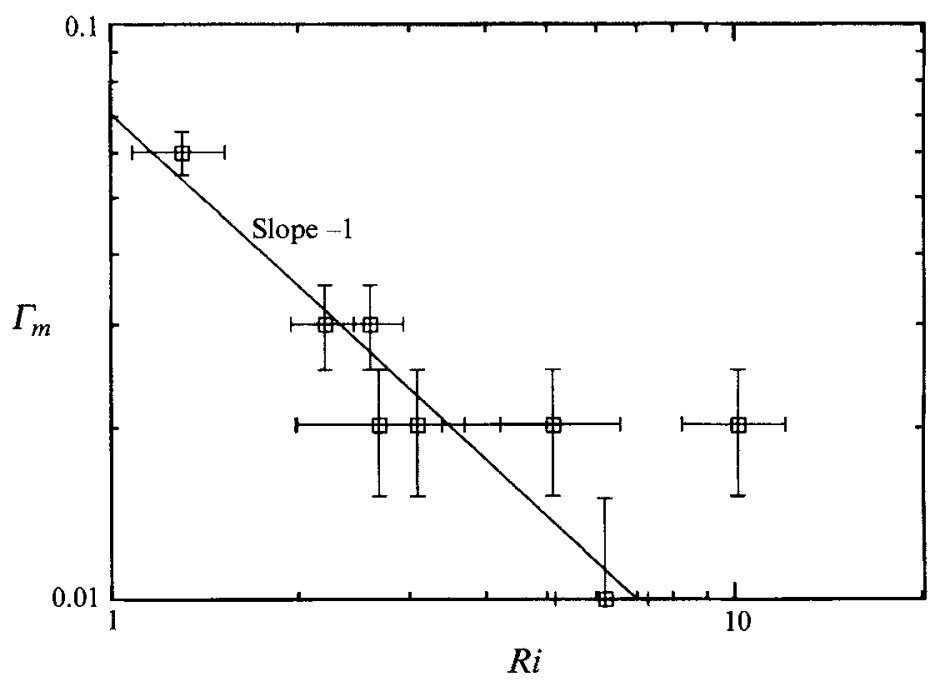

FIGURE 8. Maximum concentration anomaly for probability threshold, $K=0.01$.

maximum concentration anomaly of fluid scoured from the interface and permanently entrained. In the $R i=4.9$ experiment, the amplitudes of the interfacial instabilities are large enough to contribute to the p.d.f. a distance $\frac{1}{2} h_{1}$ above the interface; fluid raised a distance $\frac{1}{2} h_{1}$ from the interface by an interfacial instability typically falls back to the interface and is not incorporated in the entraining layer. Therefore, $\Gamma_{m}$ at $R i=4.9$ is not included in figure 8 . The model developed in $\S 4.1$ predicts $\Gamma_{m} \sim R i^{-1}$. With the exception of one point at $R i=10.1$ (whose error may be somewhat underestimated), the data are consistent with the model prediction.

Power-spectral-density estimates for concentration fluctuations are shown in figure 9. These are determined using a slightly modified version of a method suggested by Press et al. (1989). Here the concentration power spectral density is defined such that

$$
\frac{\overline{c^{\prime 2}}}{(\Delta C)^{2}}=\int_{0}^{\infty} E_{c}(f) \mathrm{d} f
$$

where $E_{c}$ is the concentration power spectral density and $f$ is the frequency. In figure 9 , normalized spectra, $E_{c} u / \overline{c^{\prime 2}} h$, are plotted versus normalized frequency, $f h / u$. Here $u$ is the mean streamwise velocity measured a distance $\frac{1}{2} h_{1}$ above the interface, $c^{\prime}$ is the local (normalized) r.m.s. concentration, and $h$ is the concentration-boundary-layer thickness. The spectra collapse quite well using this inertial (large-scale) scaling, which suggests that large-scale disturbances scale with $h$ and convect with the local mean velocity, $u$, independent of $R i$.

Mean interface thicknesses, $\bar{\delta}$, are shown versus $R i_{s}$ in figure 10 . The interfacial thickness, $\delta(t)$, is defined as the vertical distance between the point below the interface centre where $c$ first rises above $0.9 \Delta C$, and the point above the interface centre where $c$ first drops below $0.1 \Delta C$. The model in $\S 4$ predicts $\bar{\delta} / h_{s} \sim R i_{s}^{-1}$ in the $\mathrm{K}$-H-dominated regime $\dagger$ and

$$
\bar{\delta} \sim \frac{\left(U_{0} / R i_{0}\right)^{1 / 2}+\left(U_{1} / R i_{1}\right)^{1 / 2}}{\left(U_{0} / h_{0}\right)+\left(U_{1} / h_{1}\right)}
$$

$\dagger$ The velocity gradient thickness, $h_{s}$, is introduced to show the differing forms for $\delta$ in the two mixing regimes. 


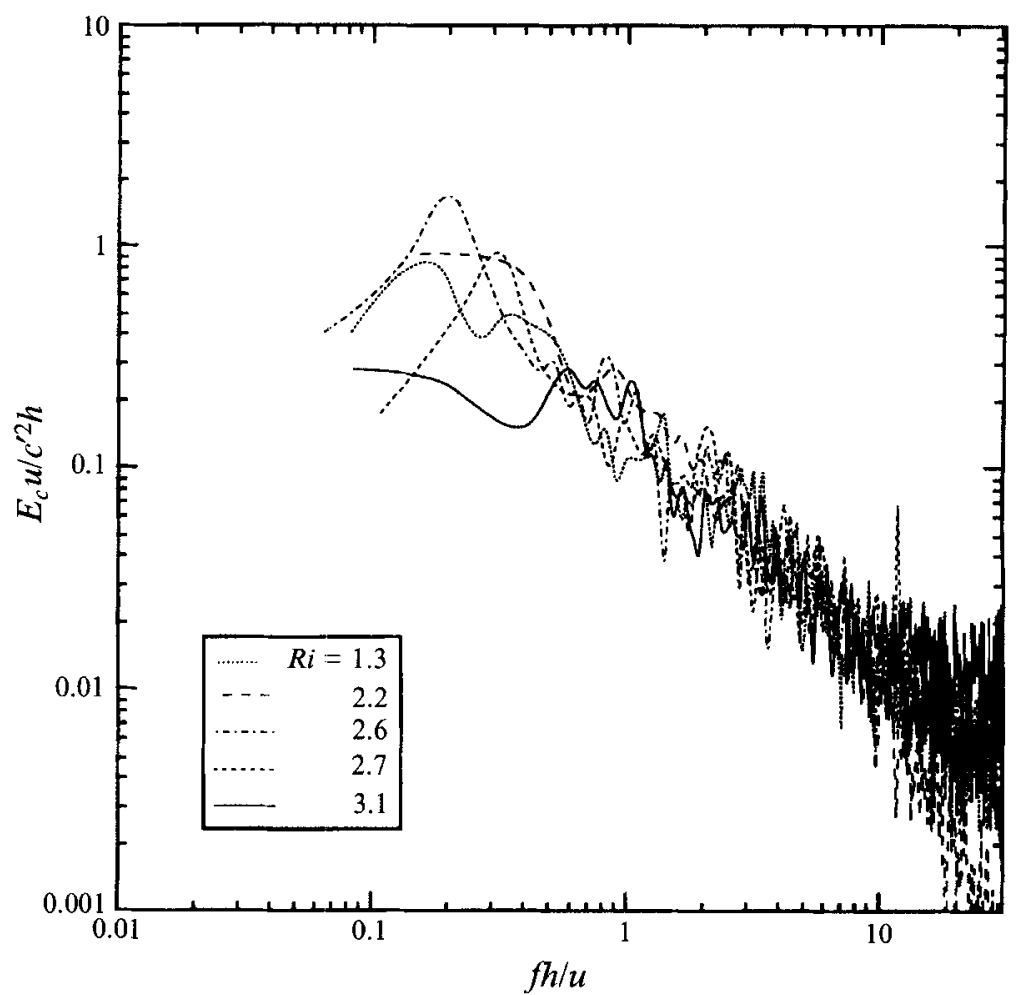

FIGURE 9. Power spectral density estimates for concentration. Estimates are from the upper layer at $(y-\eta) / h_{1}=0.5$.

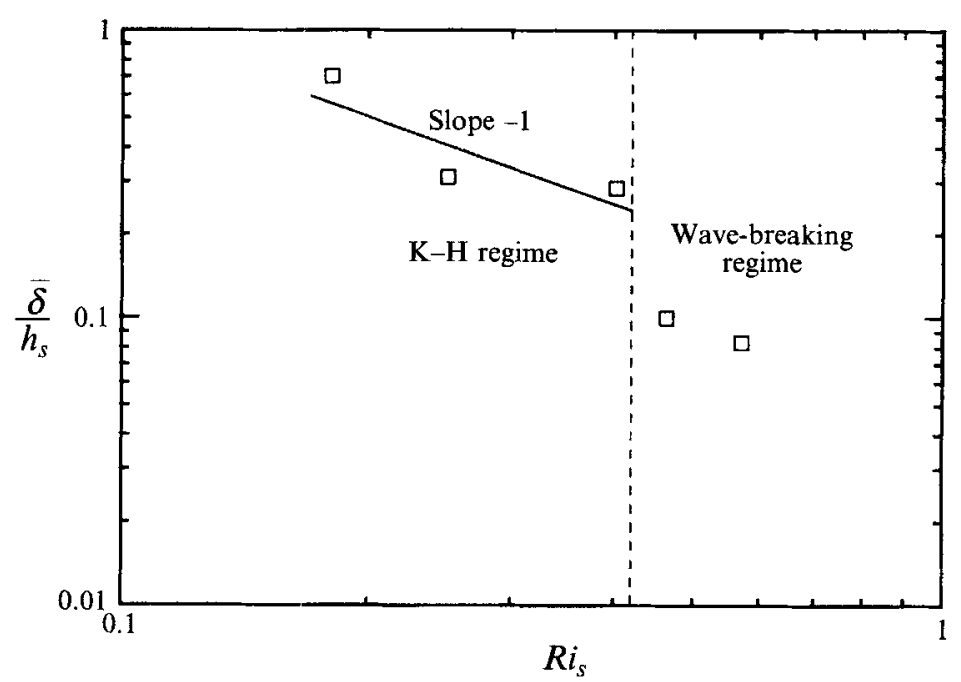

Figure 10. Mean interface thickness. 
in the wave-breaking-dominated regime. Although admittedly sparse, the data shown are not consistent with the model results.

\section{Entrainment model}

Motivated by experimental findings reported in this paper and in the companion paper, Sullivan \& List (1993), a model for interfacial mixing and vertical transport is developed.

\subsection{Model development}

Consider two fluid layers of density $\rho_{1}$ and $\rho_{1}+\Delta \rho$, with free-stream velocity $U_{1}$ and $U_{0}$, and with boundary-layer thickness $h_{1}$ and $h_{0}$, respectively, separated by an interface of mean thickness $\delta$, as shown in figure 11. The layer Reynolds numbers are assumed sufficiently large that the flow is everywhere turbulent, except very close to the interface, where buoyancy forces are appreciable. Consider turbulent eddies of size $\sim \ell$ and with fluctuating velocity scale $\sim u$ advecting past the interface with mean velocity $\sim U$. (An 'eddy' is loosely defined here as a compact, coherent turbulent formation (inhomogeneity). It is assumed that large-scale eddies are responsible for the bulk of the entrainment and henceforth, unless specified otherwise, 'eddy' refers to a large-scale eddy.) The average spacing between entraining eddies is assumed to scale with the eddy size $\sim \ell$. (Here and throughout the symbol $\sim$ denotes proportionality; distributions about characteristic scales are implied, and it is assumed, at worst, that the distributions depend only weakly on $R i, R e$, or $P e$.)

It is assumed in the present model that both the vertical and horizontal turbulent velocity scales $\sim \mathscr{u}$, and are determined solely by properties of the turbulence in the bounding layers. Estimates of the equivalent Monin-Obukhov lengthscale in the turbulent layers are relatively large (Sullivan \& List 1993), suggesting that properties of the turbulence are relatively uninfluenced by the interfacial buoyancy flux (and $R i$ ). (Results for $u^{\prime} / U$ and $v^{\prime} / U$, the normalized horizontal and vertical velocity fluctuations, respectively, from Sullivan \& List 1993 also display no obvious $R i$ trends.)

The time of interaction of an eddy with the interface is assumed determined by the characteristic large-time scale of the turbulence (eddy turnover time),

$$
t_{e} \sim \ell / u \text {. }
$$

During an interaction time, $t_{e}$, an eddy, advecting horizontally with velocity $\sim U$, scours fluid over a horizontal distance $\sim U \ell / u$ along the interface. At a fixed region near the interface, the duration of influence of a horizontally propagating eddy is given by the eddy passage time,

$$
t_{p} \sim \ell / U
$$

Assuming that an eddy penetrates the interface vertically with velocity $\sim u$, the influence of a passing eddy should extend a distance into the interface, $d_{i} \sim u_{p}$; using (4.2) this gives

$$
d_{i} \sim u \ell / U
$$

From conservation of energy it is required that the kinetic energy of an eddy be greater than or equal to the work done in lifting interfacial fluid. This requires $R i<c$, where $c$ is a constant between about 10 and 100 . If $R i>c$, then the spacing between entraining eddies and the distance over which they scour may depend on $R i$. In the present analysis it is assumed that $R i$ is always less than $c$.

It is assumed that interfacial fluid is permanently entrained if it resides in the turbulent layer long enough to become 'incorporated', the incorporation process 


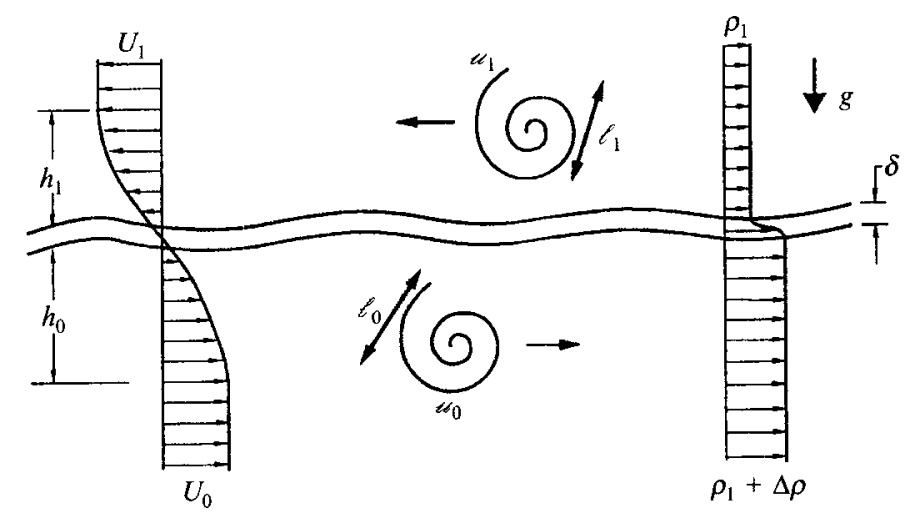

FIGURE 11. Schematic of two-layer entraining flow.

consisting of stretching fluid elements into fine strands so that molecular diffusion can act effectively. Broadwell \& Mungal (1991) suggest that the time required to stretch the interface of a large-scale 'blob' of scalar, to scales on the order of the Kolmogorov microscale, scales with $\ell / u$. The additional time required for local straining to further reduce the scale of the interface (to the Batchelor scale) and for subsequent molecular diffusion is negligible compared with $\ell / u$, so that the total time for 'incorporation' scales with $\ell / \varkappa$. Scoured interfacial fluid whose residence time is less than this falls back to the interface and is not entrained.

Consider an element of fluid of density $\rho_{1}+\theta \Delta \rho(\theta$ ranging from 0 to 1$)$ scoured from the interface and accelerated to velocity $\sim u$ in the vertical. Upon being scoured, the fluid element is subject to a gravitational force (per unit volume) $\sim \Gamma \Delta \rho g$ acting in a direction toward the interface. Here $\Gamma$ is the concentration anomaly, defined such that if fluid is scoured from above, $\Gamma=\theta$, and if fluid is scoured from below, $\Gamma=1-\theta$. In the absence of additional forces, a simple dynamical argument implies that the fluid element resides in the turbulent layer for a time

$$
t_{a} \sim 2 u /(\Gamma \Delta b)
$$

For permanent entrainment of this element it is necessary that $t_{a}$ be greater than the time for interaction to allow incorporation $\left(t_{e} \sim \ell / \varepsilon\right)$, thus implying an upper bound on $\Gamma$ for permanent entrainment, given by

$$
\Gamma_{m} \sim \frac{2 u^{2}}{\Delta b \ell} .
$$

Now consider a stretch of interface along which $n$ eddies from one adjacent layer scour fluid from the interface. The one-way entrainment velocity, $u_{e}$, is defined here as the equivalent volume of adjacent layer fluid incorporated into an entraining layer per unit area per unit time. Thus, the net volume increase per unit area per unit time in the upper layer is $u_{e 1}-u_{e 0}$, and the net gain of stratifying scalar per unit area per unit time is $u_{e 1} C_{0}-u_{e 0} C_{1}$. (If $u_{e}$ were simply defined as the volume of fluid scoured from the interface by an entraining layer, then account would have to be taken both of the volume lost by the entraining layer back to the interfacial region by interfacial instabilities and by molecular diffusion, and the distribution of concentration entrained from the interfacial region.) In the regime of flow under consideration, colour flow visualizations (figure $5 a-e$ ) make it clear that fluid is never directly entrained from one layer to the other; entrainment is always of intermediate-density fluid from the 
interfacial region. (For convenience, $u_{e}$ will henceforth denote the one-way entrainment velocity from the lower to upper layer, and all unsubscripted symbols will refer to upper-layer variables.) In an interaction time $\sim t_{e}$, the $n$ eddies, which occupy a distance $\sim n \ell$, will scour fluid over a total effective distance $\sim n U \ell / u$. The one-way entrainment velocity is then given by the volume of fluid drawn from the interfacial region (corrected by a scaling factor, $\Gamma$, which accounts for the fraction of entraininglayer fluid already present in the scoured interfacial fluid) divided by the interfacial area and interaction time; i.e.

$$
u_{e} \sim \frac{n(U \ell / u)}{n \ell(\ell / u)} \int_{0}^{a_{m}} \Gamma(\xi) \mathrm{d} \xi,
$$

where $d_{m}$ is the maximum depth into the interface from which fluid may be drawn and permanently entrained. (Note that the interaction time, $t_{e} \sim \ell / \mu$, appears in the numerator and denominator of (4.6); the model result (4.6) in effect does not critically depend on the choice of this parameter.) Consider that $\Gamma$ is a slowly varying function, except in a very narrow region near the centre of the interface, e.g.

$$
\Gamma(\xi)=2^{n-1}(\xi / \delta)^{n}
$$

for $0 \leqslant \xi \leqslant \frac{1}{2} \delta$, where $\xi$ is distance from the outer edge of the interface (positive in a direction toward the centre of the interface), and $n \gg 1$. (The interfacial concentration profiles shown in figure 4 at $R i_{s}=0.46$ and 0.57 have this general appearance; the profiles shown at $R i_{s}=0.18,0.25$, and 0.40 appear broadened because of the averaging process over $\mathrm{K}-\mathrm{H}$ interfacial instabilities.) It then follows that

$$
d_{m}=\frac{1}{2} \delta\left(2 \Gamma_{m}\right)^{1 / n} .
$$

Substituting (4.8) and (4.7) into (4.6) gives

$$
\frac{u_{e}}{U} \sim \frac{\delta}{\ell}\left[\frac{2^{1 / n}}{2(n+1)}\right]\left(\Gamma_{m}\right)^{1+1 / n}
$$

Since $n \gg 1$ (the core region where concentration varies rapidly is very narrow) (4.8) may be given approximately by

and (4.9) by

$$
d_{m} \sim \delta,
$$

$$
\frac{u_{e}}{U} \sim \frac{\delta}{\ell} \Gamma_{m}
$$

(Of course a weak dependence of $d_{m}$ on $R i$ is admitted in (4.10) and leads to a slightly modified $\Gamma_{m}$ exponent in (4.11), but to a first approximation these are neglected in the present analysis.) Substituting (4.5) into (4.11) yields

$$
E \sim \frac{\delta}{\ell}\left[\frac{\mu^{2}}{\Delta b \ell}\right],
$$

where $E=u_{e} / U$ is the normalized entrainment velocity. (If the interface velocity, $u_{i}$, is non-zero, then $U$ must be replaced by $\left|U-u_{i}\right|$.)

It then remains to find $\delta / \ell$. In a local equilibrium state, the mean interface thickness, $\delta$, is determined by a balance between eddy scouring (interface sharpening) and interfacial instabilities and molecular diffusion (interface thickening). Three interfacial mixing regimes are now considered: (i) molecular diffusion dominated, (ii) wave breaking dominated, and (iii) Kelvin-Helmholtz $(\mathrm{K}-\mathrm{H})$ dominated. 


\section{Case 1: molecular-diffusion-dominated regime}

When the stability of the interface is so great that interfacial instabilities rarely, if ever, occur, then the thickening of the interface is due primarily to molecular diffusion. In this case, the rate of thickening of the interface (in the absence of eddy scouring) is $\mathrm{d} \delta / \mathrm{d} t \sim \kappa / \delta$, where $\kappa$ is the molecular diffusivity of the stratifying scalar. The volume gained per unit area per unit time by the interface is then

$$
u_{D} \sim \kappa / \delta \text {. }
$$

(It is implicitly assumed in (4.13) that the area of the distorted interface is not significantly greater than its projection on the horizontal plane.)

The volume lost from the interface per unit area per unit time, due to eddy scouring, is given by

Using (4.10), this gives

$$
u_{L} \sim d_{m 0} U_{0} / \ell_{0}+d_{m 1} U_{1} / \ell_{1} .
$$

$$
u_{L} \sim \delta\left[\frac{U_{0}}{\ell_{0}}+\frac{U_{1}}{\ell_{1}}\right] .
$$

Assuming an equilibrium state in which thickening and scouring are matched, $u_{D} \sim u_{L}$, the mean interface thickness is then

$$
\delta_{D} \sim \frac{\kappa^{1 / 2}}{\left[\frac{U_{0}}{\ell_{0}}+\frac{U_{1}}{\ell_{1}}\right]^{1 / 2}} .
$$

It is assumed now that the eddy lengthscale, $\ell$, scales with the turbulent-layer thickness, $h$. This is supported by experimental observations which suggest that the eddy passage time $\sim h / U$ is independent of $R i$, and by the observed collapse of the concentration spectra in figure 9 using $h$ as the scaling length. It is also assumed that the turbulence velocity scale, $u$, scales with the mean velocity $U$. This follows from assuming $u \sim u_{*}$, where $u_{*}=\left(\tau_{i} / \rho\right)^{1 / 2}$ is the interfacial shear velocity and $\tau_{i}$ is the interfacial shear stress, and assuming that $u_{*} / U$ does not vary appreciably with $R i, R e$, or $P$ e. (Normalized velocity fluctuations reported in Sullivan \& List 1993 show no obvious $R i$ trends, nor do results for $u^{\prime} / U$ from Stephenson \& Fernando 1991; of course a weak dependence of $u_{*} / U$ on $R i$, $R e$, or $P e$ may exist, but as a first approximation $u_{*} / U$ is taken here as constant.) Substituting (4.16) into (4.12), and assuming $\ell \sim h$ and $u \sim U$, then gives

$$
E \sim \frac{R i^{-1}}{\left[\left(h^{2} / h_{0}^{2}\right) P e_{0}+\left(h^{2} / h_{1}^{2}\right) P e_{1}\right]^{1 / 2}} .
$$

When fluid on only one side of the interface is turbulent and entraining, this reduces to

$$
E \sim R i^{-1} P e^{-1 / 2} .
$$

Case 2: wave-breaking-dominated regime

When the stability of the interface is such that large-scale $\mathrm{K}-\mathrm{H}$ instabilities do not occur, but finite-amplitude waves generated by turbulence-induced pressure fluctuations become gravitationally unstable (upon interacting with the mean shear) and break, then the thickening of the interface is dominated by wave breaking.

It may be assumed that waves generated with sufficiently large aspect ratio, $a / \lambda$ ( $a$ is the initial wave amplitude and $\lambda$ is its wavelength), are distorted relatively quickly 
by the mean shear; the wave amplitudes and wavelengths of these waves at the time of breaking should not be significantly different from their initial values (Frankignoul 1972). If an eddy generates a disturbance at the interface of wavelength $\lambda \sim U \ell / u$, with energy proportional to the kinetic energy of the eddy, then the wave amplitude, $a \sim\left(\ell u^{3} / \Delta b U\right)^{1 / 2}$. This is supported by experimental results from Narimousa \& Fernando (1987). Assuming the frequency of wave breaking scales with the frequency of wave generation $\sim U / \ell$, the volume gained per unit area per unit time by the interface is

$$
u_{B} \sim\left(u_{0}^{3} U_{0} / \Delta b \ell_{0}\right)^{1 / 2}+\left(u_{1}^{3} U_{1} / \Delta b \ell_{1}\right)^{1 / 2}
$$

Again, assuming an equilibrium state, $u_{B} \sim u_{L}$, the mean interface thickness is

$$
\delta_{B} \sim \frac{\left(\varkappa_{0}^{3} U_{0} / \Delta b \ell_{0}\right)^{1 / 2}+\left(u_{1}^{3} U_{1} / \Delta b \ell_{1}\right)^{1 / 2}}{U_{0} / \ell_{0}+U_{1} / \ell_{1}} .
$$

Substituting (4.20) into (4.12), and assuming, as before, $\ell \sim h$ and $\varkappa \sim U$, yields

$$
E \sim \frac{\frac{U_{0}}{R i_{0}^{1 / 2}}+\frac{U_{1}}{R i_{1}^{1 / 2}}}{h\left[U_{0} / h_{0}+U_{1} / h_{1}\right]} R i^{-1}
$$

When fluid on only one side of the interface is turbulent and entraining, this reduces to

$$
E \sim R i^{-3 / 2}
$$

\section{Case 3: $K$-H-dominated regime}

When the stability of the interface is such that large-scale $\mathrm{K}-\mathrm{H}$ instabilities occur, then these dominate interface thickening. Interfacial mixing (thickening) is effected by tubulent billows generated by the roll-up of $\mathrm{K}-\mathbf{H}$ instabilities. The maximum amplitude, $a_{B}$, achieved by a billow, before buoyancy forces precipitate its collapse, is given by

$$
a_{B} \sim(\Delta U)^{2} / \Delta b,
$$

as suggested by the experiments of Thorpe (1973), where $\Delta U$ is the velocity difference between the two layers. After billow collapse, the time required for turbulent eddies to resharpen the interface for a successive instability is

$$
t_{s} \sim \delta_{K} / u_{L}
$$

and the volume gained per unit area per unit time by the interface is

$$
u_{K} \sim \frac{(\Delta U)^{2}}{\Delta b \delta_{K}} u_{L}
$$

(It is implicitly assumed in (4.25) that the time for a billow to form and collapse is considerably less than the time for eddies to resharpen the interface.)

As before, an equilibrium state in which $u_{K} \sim u_{L}$ implies that the mean interface thickness is

$$
\delta_{K} \sim(\Delta U)^{2} / \Delta b
$$

Substituting (4.26) into (4.12), and assuming, as before, $\ell \sim h$ and $\varkappa \sim U$, yields

$$
E \sim R i^{-1}\left[\frac{(\Delta U)^{2}}{\Delta b h}\right]
$$


When fluid on only one side of the interface is turbulent and entraining, it may be assumed that $\Delta U \sim U$, and (4.27) reduces to

$$
E \sim R i^{-2} .
$$

\subsection{Energy considerations}

When fluid on only one side of the interface is turbulent and entraining, the rate of supply of energy to the interfacial region (per unit area) by the turbulent eddies is

$$
e_{s} \sim \rho u^{3} .
$$

The concomitant rate of gain of potential energy by the entraining layer (per unit area) is

$$
e_{p} \sim \Delta \rho g u_{e} \ell
$$

The ratio of these, $\beta=e_{p} / e_{s}$, represents the fraction of eddy kinetic energy that is converted to potential energy, or 'efficiency' of energy conversion. Assuming $\ell \sim h$ and $u \sim U$, gives

$$
\beta \sim R i E .
$$

In the diffusion-dominated regime, $E \sim R i^{-1} P e^{-1 / 2},(4.18)$, and

$$
\beta \sim P e^{-1 / 2} .
$$

As $P e$ decreases, $\delta / \ell$ increases, (4.16), and an increasing fraction of eddy kinetic energy goes toward raising fluid for permanent entrainment. (This does not violate the assumption of proportionality between wave energy and eddy kinetic energy, however, since it is assumed that $\beta$ is always small.) To maximize entrainment, energy transferred to interfacial waves should be minimized, since this energy is lost to viscous dissipation and to the mean flow through shear-wave interaction.

In the wave-breaking-dominated regime, $E \sim R i^{-3 / 2},(4.22)$, and

$$
\beta \sim R i^{-1 / 2} \text {. }
$$

Although a portion of energy supplied to interfacial waves goes toward increasing $\delta / \ell$ (and augmenting entrainment), a fraction of wave energy is transferred back to the mean flow through shear-wave interaction and is dissipated in the wave breaking process. As $R i$ decreases, the energy lost (eventually) to dissipation and to shear-wave interaction decreases (relatively), and the potential energy gain by the entraining layer increases.

In the $\mathrm{K}-\mathrm{H}$-dominated regime, $E \sim R i^{-2},(4.28)$, and

$$
\beta \sim R i^{-1} \text {. }
$$

$\mathrm{K}-\mathrm{H}$ billows, which extract energy from the mean flow directly, dominate the thickening process, so that energy transferred to interfacial waves contributes little to $e_{p}$. As $R i$ decreases, $\delta / h$ increases, (4.26), and a greater fraction of fluid scoured from the interface is permanently entrained.

\subsection{Mixing regime boundaries}

Boundaries separating mixing regimes are now explored for the case when one side of the interface is turbulent and entraining. As discussed in $\$ 3$, it appears that (cyclically occurring) $\mathrm{K}-\mathrm{H}$ instabilities are active when $R i_{s}$ is less than about $0.4-0.45$. Assuming $R i_{s}=R i_{s}(R i)$, the upper bound on $R i$ for (cyclical) $\mathrm{K}-\mathrm{H}$ instability may be taken as $R i=R i_{c}$ (Narimousa \& Fernando 1987 suggest $R i_{c} \sim 5$ ). The lower bound for $R i$ in the proposed $\mathrm{K}-\mathrm{H}$-dominated regime is governed by the onset of small-scale turbulence in the interface, occurring at, say, $R i=R i_{t}$. In the wave-breaking-dominated regime, 


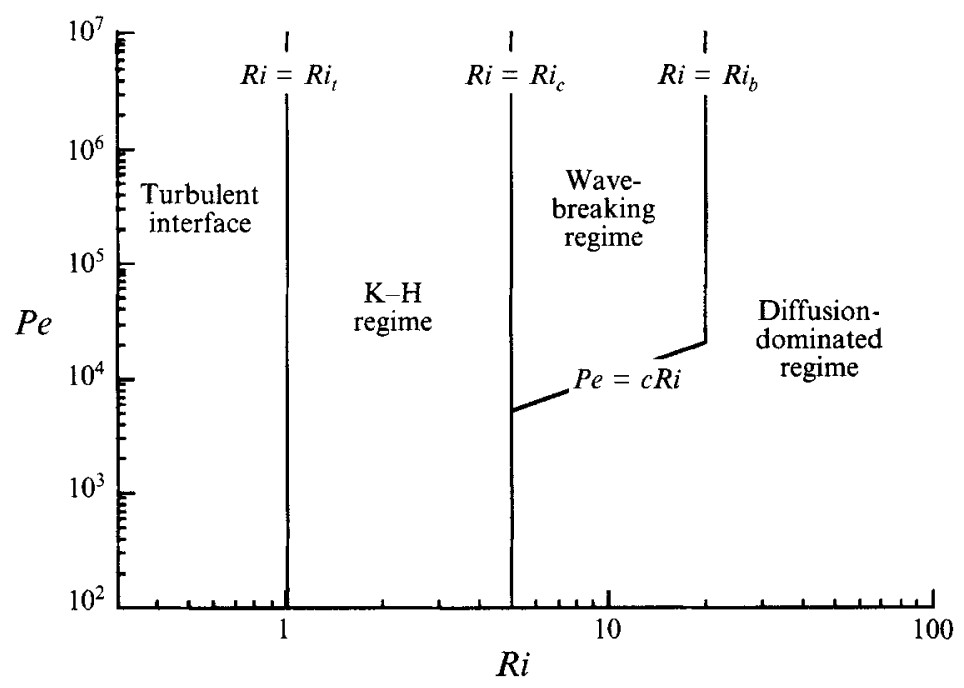

FIGURE 12. Mixing-regime boundaries in $R i-P e$ space for the case when a single layer is turbulent and entraining. Numerical values of $R i$ and $P e$ delineating borders are only approximate.

mixing by (shear-driven) wave instabilities is effective when $a / \lambda>(a / \lambda)_{c}$, setting an upper bound on $R i$ for effective wave breaking, given by $R i_{b} \sim\left[(a / \lambda)_{c}\right]^{2}$ (Narimousa \& Fernando suggest $R i_{b} \sim 20$ ). When $R i_{c}<R i<R i_{b}$, interfacial thickening by molecular diffusion is also active. Using (4.13), (4.15), and (4.19), its contribution becomes important (relative to mixing by wave breaking) when

$$
P e=c R i,
$$

where $c$ is a constant of order 1000 .

Proposed boundaries separating mixing regimes for the single-layer entrainment case are shown in figure 12 . When both layers are turbulent and entraining, the delineation of mixing regime boundaries is considerably more difficult: $R i_{s}$ is no longer determined by a single-layer $R i$, nor is the onset of turbulence in the interface. Details of the local velocity field dependence on governing parameters are required, which is beyond the scope of the present study.

\subsection{Comparison with experiment}

Before comparing model predictions to experimental results, it should be noted that there is an inherent difficulty in fitting power laws to experimental data over narrow ranges of a governing parameter. In the following discussion, it should be kept in mind that the power law fits to the data are not definitive; rather, they demonstrate that the data are consistent with the present model predictions.

Experimental results from the present study lend support to the model predictions. As seen in figure 8, the data for $\Gamma_{m}$ are consistent with $\Gamma_{m} \sim R i^{-1}$, independent of the dominant interfacial mixing mechanism, as predicted by the model. In figure 10 , the three measurements of interfacial thicknesses in the $\mathrm{K}-\mathrm{H}$-dominated regime are consistent with the model result, $\delta \sim c_{1}(\Delta U)^{2} / \Delta b$, with $c_{1} \sim 0.1$. In the wave-breakingdominated regime, two measurements of interfacial thicknesses are consistent with the model result, (3.6), with constant of proportionality $\sim 0.4$.

Results from previous studies lend some further support to the proposed entrainment model. (Care must be taken, however, in assessing numerical values of $E$ and $R i$ from different experiments, as different investigators use different velocity and length scales.) 


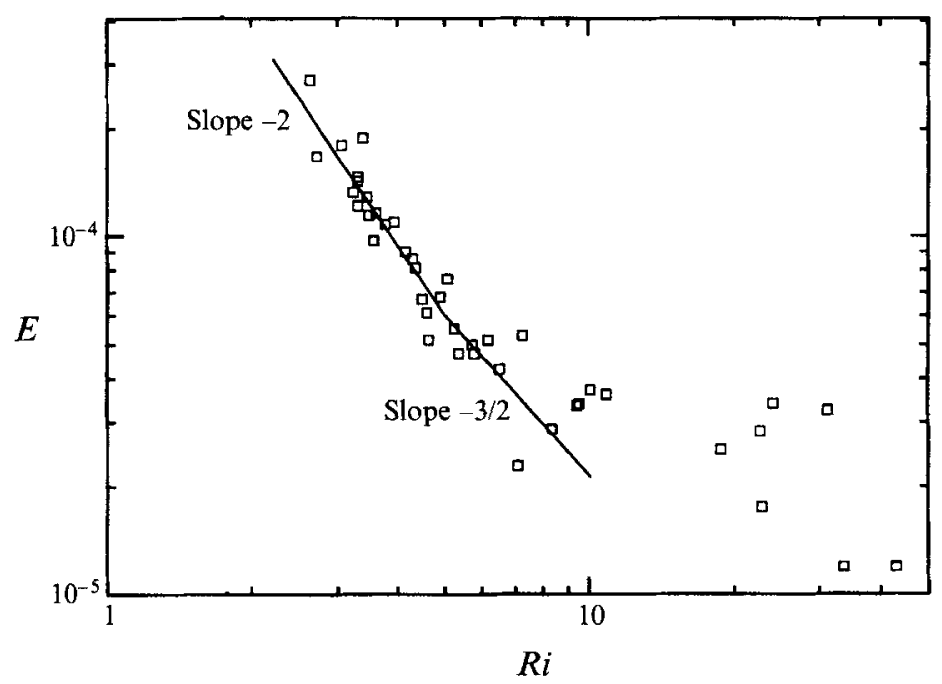

FIGURE 13. Entrainment results from Lofquist (1960). $R i$ is based on the mean layer velocity and the layer hydraulic radius (typically about one-third of the layer depth).

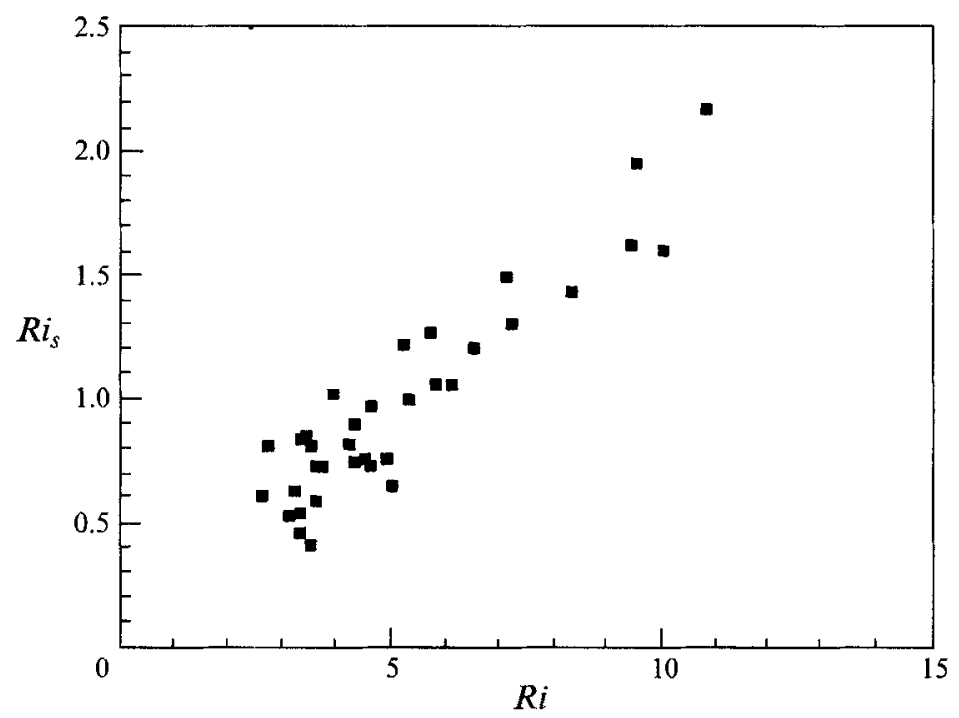

FiguRE 14. Estimates of $R i_{s}$ from Lofquist (1960).

Entrainment results from Lofquist (1960) are re-plotted in figure 13. They may be interpreted as $E \sim R i^{-2}$ for $R i$ less than about 5 , and $E \sim R i^{-3 / 2}$ for $5<R i<10$, consistent with model predictions (assuming, in this case, that mixing is $\mathrm{K}-\mathrm{H}$ dominated for $R i<5$, and wave breaking dominated for $5<R i<10$ ). For $R i>10$ the data are widely scattered owing to experimental difficulties associated with measuring low entrainment rates. (It is also expected that diffusion effects become important at high $R i$.)

In figure 14 estimates of $R i_{s}$ from Lofquist are plotted versus $R i$. Here $R i=5$ corresponds to a value of $R i_{s}$ of about 1, considerably greater than the $0.4-0.45$ range for transition between $\mathrm{K}-\mathrm{H}$-dominated and wave-breaking-dominated mixing found in the present study. In Lofquist's experiments a pendulum force balance apparatus 


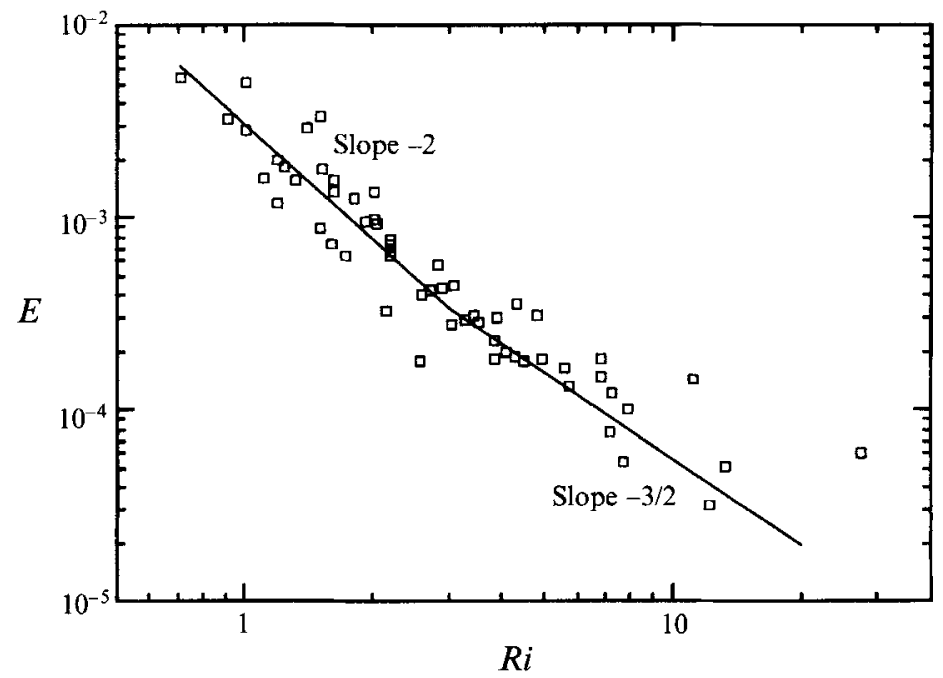

FiguRE 15. Entrainment results from Deardorff \& Willis (1982). Ri is based on the mixed layer depth and the velocity jump at the interface.

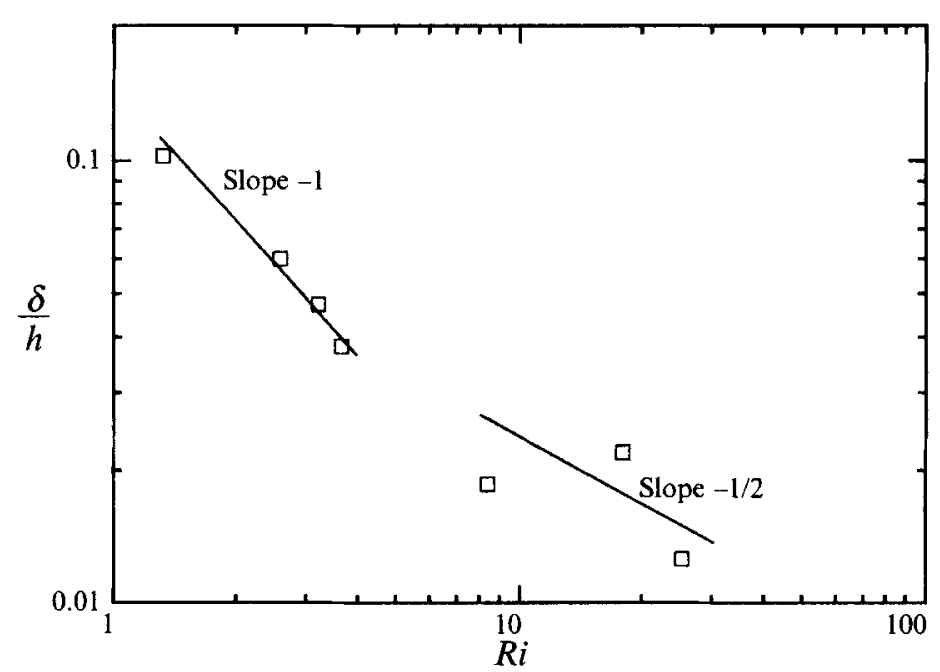

FiguRE 16. Mean interface thicknesses from Stephenson \& Fernando (1991). $R i$ is based on the turbulent layer depth and layer mean velocity.

was employed to measure mean velocity. The inherent difficulty in measuring the maximum velocity gradient at the interface with this technique may account for the differing values of $R i_{s}$ found in Lofquist's experiments compared with those in the present study.

Entrainment results from Deardorff \& Willis (1982) are re-plotted in figure 15. Their results may be interpreted as $E \sim R i^{-2}$ for $0.7<R i<3$ and $E \sim R i^{-3 / 2}$ for $3<R i<$ 20 , consistent with model predictions (assuming, in their case, that mixing is $\mathbf{K}-\mathbf{H}$ dominated in the first range and wave breaking dominated in the second).

Recent measurements of interfacial thicknesses using LIF by Stephenson \& Fernando (1991) are re-plotted in figure 16. For $R i$ less than about 5, the data are well represented by $\delta / h \sim R i^{-1}$, consistent with the model result (assuming mixing is $\mathrm{K}-\mathrm{H}$ dominated). 
For $R i>5$, their results are consistent with $\delta / h \sim R i^{-1 / 2}$; however, scatter in the data is appreciable.

\section{Discussion}

Although the present model predictions receive support from this study's experiments, and from the work of Lofquist (1960), Deardorff \& Willis (1982), and Stephenson \& Fernando (1991), some seemingly contradictory results appear in the literature, which must be reconciled.

As mentioned in $\S 1$, Ellison \& Turner (1959) studied entrainment primarily over a range of $R i, 0<R i<0.8$, for which the interface was turbulent. In this case, adjacentlayer fluid is directly incorporated by the engulfing action of locally generated eddies, rendering the present model analysis invalid. Any similarity in form of results to present model predictions for quasi-laminar entrainment would be entirely fortuitous.

Although the entrainment results of Lofquist lend support to the current model, his interface measurements suggest $\delta / h \sim$ constant for $2<R i<40$, at variance with present model predictions, and in disagreement with experimental results of the present work and of Stephenson \& Fernando. Narimousa \& Fernando (1987) also suggest $\delta / h \sim$ constant for $1<R i<15$. A lack of spatial resolution in both Lofquist's and Narimousa \& Fernando's experiments may explain the apparent discrepancy, since interfaces measured with high-resolution instrumentation, both in this study and by Stephenson \& Fernando, are considerably thinner than those perceived by Lofquist and Narimousa \& Fernando.

Kato \& Phillips (1969) and Kantha et al. (1977) used the shear velocity, $u_{*}$, to scale their results. Since the contribution to $u_{*}$ from sidewall drag can be appreciable in their experiments (Price 1979), it is difficult to compare directly their results with predictions of the present entrainment model. Price (1979) attempted to re-scale their results using an inferred mean velocity; however, his analysis is questionable (Deardorff \& Willis 1982), and the derived result, $E \sim R i^{-4}$, is somewhat dubious.

Results of Moore \& Long (1971) suggest an entrainment relation $E \sim R i^{-1}$ for $1<R i<30$, in disagreement both with present model predictions and with results from Lofquist and Deardorff \& Willis. Linden (1973) comments that jets employed by Moore \& Long to generate their flow must introduce additional turbulent length and velocity scales, not accounted for in a single $R i$ parameterization. The extent to which the jets influence entrainment at the interface in their experiments is uncertain, and the relevance of Moore \& Long's result is debatable.

More recent results from Narimousa \& Fernando appear to follow $E \sim R i^{-1.2}$, for $1.5<R i<15$, also at variance with the present model predictions and with results from Lofquist and Deardorff \& Willis. The reason for this discrepancy is currently not understood. It is possible that peculiarities in start-up conditions may account for this; however, more experimental information is necessary to resolve the definite cause.

Although the entrainment model developed by Linden (1973) pertains, strictly speaking, to zero-mean-shear entrainment, it is instructive to compare his model to that developed here. In his model, Linden assumes that large-scale eddies are responsible for the bulk of entrainment and models them as a collection of coherent vortices. He assumes that the eddies, or vortices, randomly impinge on the interface and that entrainment is by ejection ('splashing') of adjacent-layer fluid into the turbulent layer during interface recoil (as suggested by his experiments using vortex rings). He assumes that potential energy gained through entrainment is proportional to kinetic energy supplied by impinging eddies. He further assumes that the spacing 
between impinging eddies is proportional to the distorted eddy size, $\ell^{\prime} \sim \ell R i^{1 / 2}$, and that the vortex interaction time scales with $(\ell / \Delta b)^{1 / 2}$ (interface recoil time). From this, he derives $E \sim R i^{-3 / 2}, R i$ being defined in terms of characteristic eddy velocity and length scales.

In the current model, large-scale eddies are also responsible for the bulk of entrainment. However, the eddies cannot fully penetrate the interface and permanently entrain adjacent-layer fluid; rather, they can only scour pre-mixed intermediatedensity fluid from the interfacial region. The present model is restricted to flows in which the interfacial region is quasi-laminar, i.e. high- $R$ i flows; the eddies in this case do not posses sufficient energy to raise fluid from the adjacent layer for permanent entrainment. In Linden's vortex ring experiments, the effective Richardson number varies between about 1.5 and 50 , based on the characteristic velocity and length scales of the eddies (vortices). The equivalent range of $R i$ in the present experiments is approximately 100-1000. In Linden's model, the eddies are more energetic and posses sufficient energy to entrain fluid directly from the adjacent layer.

As in Linden's model, energy transferred to interfacial disturbances in the present model is proportional to kinetic energy supplied by large-scale eddies. However, in contrast with Linden's model, potential energy ultimately gained by the system is not proportional to energy supplied by the eddies. A fraction of interfacial disturbance energy (not proportional to eddy kinetic energy) is lost through viscous dissipation and is transferred back to the mean flow through shear-wave interaction.

In Linden's model, the eddy-interface interaction process is governed by buoyancy forces at the interface. In his model, the rate of supply of energy to the interface by turbulent eddies is given by $\rho \varkappa^{3} R i^{-1 / 2}$ per unit area. In the present model, the mutual interaction between eddies in the bounding turbulent layers determines the rate of supply of energy to the interfacial region, independent of the buoyancy jump at the interface. This is a fundamental difference between the present model and that of Linden. The eddy-interface interaction process in the present model consists of eddies randomly impinging on the interface and scouring interfacial fluid into adjacent layers on a time scale proportional to $\ell / u$, the characteristic large-time scale of the turbulence. The spacing of entraining eddies in the present model does not depend on interfacial wave dynamics, but only on characteristics of the turbulence away from the interface. In Linden's model, it is precisely the interfacial wave dynamics that determine the interaction time of eddies with the interface and the distorted eddy size, $\ell^{\prime}$ (which determines the spacing between entraining eddies).

Linden's model is essentially a zero-mean-shear representation of entrainment in which there is no local generation of turbulence near the interface. In the present model, turbulence is locally generated by means of the local velocity shear, and it is assumed that the interaction process is governed by the shear-generated turbulence. Linden's model (presumably) was developed to describe low- $R i$ zero-mean-shear experiments; the present model pertains to shear flows involving quasi-laminar internal interfaces. The present model predicts $E \sim R i^{-1} P e^{-1 / 2}, E \sim R i^{-3 / 2}$, and $E \sim R i^{-2}$ in the diffusive, wave breaking, and $\mathrm{K}-\mathrm{H}$ mixing regimes, respectively; Linden's model predicts $E \sim R i^{-3 / 2}$. In the light of the differing model assumptions, the agreement between the two in the present model's wave breaking regime is a happenstance.

Mory (1991) has also developed a model for zero-mean-shear entrainment. In contrast to Linden's model, Mory assumes that entrainment is due to small-scale eddies which induce high shear rates locally at the interface, resulting in small-scale $\mathrm{K}-\mathrm{H}$ instabilities. He assumes that potential energy gained through $\mathrm{K}-\mathrm{H}$ mixing (and subsequent advection of mixed fluid by turbulent eddies) is proportional to kinetic 
energy supplied by small-scale eddies. From this, he derives $E \sim R i^{-3 / 2}$ for large $P e$, and $E \sim P e^{-1 / 3} R i^{-1}$ for low to moderate $P e, R i$ and $P e$ being defined in terms of local turbulent length and velocity scales.

Contrary to the Mory model, experimental observations in the present work suggest entrainment is due to large-scale eddies, and there appears to be no evidence of smallscale $\mathrm{K}-\mathrm{H}$-type instabilities induced by small-scale eddies. Moreover, the scouring events and local interfacial instabilities (either $\mathrm{K}-\mathrm{H}$ or wave breaking) appear to be uncorrelated in time.

To conclude, it is interesting to note the difference between interfacial waves generated in the zero-mean-shear experiments of Hannoun \& List (1988) and those in the present study. In the former case, it is suggested that interfacial waves, generated by random pressure fluctuations induced by turbulent eddies, induce local regions of intense shear in the interface which lead to breakdown by $\mathrm{K}-\mathrm{H}$ instability (as postulated by Phillips 1966). Wave energy is confined to a small region about the interface, and the wave field becomes saturated (to the limit of the Phillips instability) over a finite range of wavenumbers. In the present experiments, it appears that Phillips' mechanism for interfacial mixing is inactive, since waves generated by turbulent pressure fluctuations are immediately distorted by the mean shear, and either transfer energy back to the mean flow through shear-wave interaction (Phillips 1966) or, if their initial aspect ratio $(a / \lambda)$ is sufficient large, become gravitationally unstable and break in a relatively short period of time (Frankignoul 1972).

We would like to thank the entire staff of the W. M. Keck Hydraulics Laboratory for their valuable assistance throughout this study. The first author would like to thank Professor P. J. Sullivan for some valuable discussions and helpful advice and Professor H. J. S. Fernando for some useful comments. We would also like to thank Professor J. S. Turner for some thoughtful comments on an early version of the manuscript. The authors also express their appreciation for numerous comments received from anonymous reviewers, all of which materially improved the final manuscript. The majority of this work is taken from the first author's doctoral dissertation, financed in part by the US National Science Foundation through Award number CTS-8819584, by the US Department of the Interior, Geological Survey through Grant number 14-080001-G1628, through the State Water Resources Research Institute, Project number G1550, and by the University of California Water Resources Center, Project UCALWRC-W-735. Contents of this publication do not necessarily reflect the views and policies of the US Department of the Interior, nor does mention of trade names or commercial products constitute their endorsement or recommendation for use by the US Government.

\section{REFERENCES}

Broadwell, J. E. \& Mungal, M. G. 1991 Large-scale structures and molecular mixing. Phys. Fluids A 3, 1193-1206.

BRoWAND, F. K. \& WinanT, C. D. 1973 Laboratory observations of shear-layer instability in stratified fluid. Boundary-Layer Met. 5, 67-77.

DeardorfF, J. W. \& Willis, G. E. 1982 Dependence of mixed-layer entrainment on shear stress and velocity jump. J. Fluid Mech. 115, 123-149.

Ellison, T. H. \& Turner, J. S. 1959 Turbulent entrainment in stratified flows. J. Fluid Mech. 6, 423-448.

Fernando, H. J. S. 1991 Turbulent mixing in stratified fluids. Ann. Rev. Fluid Mech. 23, 455-493. 
Frankignoul, C. J. 1972 Stability of finite amplitude internal waves in a shear flow. Geophys. Fluid Dyn. 4, 91-99.

Hannoun, I. A. 1985 Matching the refractive index in density stratified fows. Tech. Mem. 85-1. W. M. Keck Laboratory of Hydraulics and Water Resources, California Institute of Technology.

Hannoun, I. A. 1987 Turbulent mixing in stably-stratified fluids subjected to zero-mean shear. PhD thesis, California Institute of Technology.

Hannoun, I. A. \& List, E. J. 1988 Turbulent mixing at a shear-free density interface. J. Fluid Mech. $189,211-234$.

Kantha, L. H., Phillips, O. M. \& Azad, R. S. 1977 On turbulent entrainment at a stable density interface. J. Fluid Mech. 79, 753-768.

Kato, H. \& PhilliPs, O. M. 1969 On the penetration of a turbulent layer into stratified fluid. J. Fluid Mech. 37, 643-655.

KOOCHESFAHANI, M. M. 1984 Experiments on turbulent mixing and chemical reactions in a liquid mixing layer. $\mathrm{PhD}$ thesis, California Institute of Technology.

Koop, C. G. \& Browand, F. K. 1979 Instability and turbulence in a stratified fluid with shear. J. Fluid Mech. 93, 135-159.

LaWrence, G. A., Browand, F. K. \& Redekopp, L. G. 1991 The stability of a sheared density interface. Phys. Fluids A 3, 2360-2370.

LINDEN, P. F. 1973 The interaction of a vortex ring with a sharp density interface: a model for turbulent entrainment. J. Fluid Mech. 60, 467-480.

LoFQuist, K. 1960 Flow and stress near an interface between stratified liquids. Phys. Fluids 3, $158-175$.

MOORE, M. J. \& LONG, R. R. 1971 An experimental investigation of turbulent stratified shearing flow. J. Fluid Mech. 49, 635-655.

MORY, M. 1991 A model of turbulent mixing across a density interface including the effect of rotation. J. Fluid Mech. 223, 193-207.

NARImousa, S. \& Fernando, H. J. S. 1987 On the sheared density interface of an entraining stratified fluid. J. Fluid Mech. 174, 1-22.

Narimousa, S., Long, R. R. \& Kitaigorodskit, S. A. 1986 Entrainment due to turbulent shear flow at the interface of a stably stratified fluid. Tellus 38A, 76-87.

Papantoniou, D. A. 1986 Observations in turbulent buoyant jets by use of laser-induced fluorescence. PhD thesis, California Institute of Technology.

PhILliPs, O. M. 1966 The Dynamics of the Upper Ocean. Cambridge University Press.

PHILlIPS, O. M. 1977 Entrainment. In Modelling and Prediction of the Upper Layers of the Ocean (ed. E. B. Kraus). Pergamon.

Press, W. H., Flannery, B. P., Teukolsky, S. A. \& Vetterling, W. T. 1989 Numerical Recipes. Cambridge University Press.

PrICE, J. F. 1979 On the scaling of stress-driven entrainment experiments. J. Fluid Mech. 90, 509-529.

RASI, M. 1989 Mixing in density-stratified conjugate flows. $\mathrm{PhD}$ thesis, California Institute of Technology.

Stephenson, P. W. \& Fernando, H. J. S. 1991 Turbulence and mixing in a stratified shear flow. Geophys. Astrophys. Fluid Dyn. 59, 147-164.

Sullivan, G. D. \& List, E. J. 1993 An experimental investigation of vertical mixing in twolayer density-stratified shear flows. Dyn. Atmos. Oceans 19, 147-174.

THORPE, S. A. 1973 Experiments on instability and turbulence in a stratified shear flow. J. Fluid Mech. 61, 731-751.

TURNER, J. S. 1968 The influence of molecular diffusivity on turbulent entrainment across a density interface. J. Fluid Mech. 33, 639-656. 